\title{
Adiabatic limit of the eta invariant over cofinite quotients of $\operatorname{PSL}(2, \mathbb{R})$
}

\author{
Paul Loya, Sergiu Moroianu and Jinsung Park
}

\begin{abstract}
The eta invariant of the Dirac operator over a non-compact cofinite quotient of $\operatorname{PSL}(2, \mathbb{R})$ is defined through a regularized trace following Melrose. It reduces to the standard definition in terms of eigenvalues in the case of a totally non-trivial spin structure. When the $S^{1}$ fibers are rescaled, the metric becomes of non-exact fibered-cusp type near the ends. We completely describe the continuous spectrum of the Dirac operator with respect to the rescaled metric and its dependence on the spin structure, and show that the adiabatic limit of the eta invariant is essentially the volume of the base hyperbolic Riemann surface with cusps, extending some of the results of Seade and Steer.
\end{abstract}

\section{Introduction}

The eta invariant was introduced by Atiyah-Patodi-Singer [APS75] as the boundary correction term in their index formula. This non-local invariant turned out to be quite elusive, although its variation is local. Motivated by physics, one successful approach to the study of the eta invariant is the so-called adiabatic limit, in which the eta invariant on the total space of a fibration is investigated when the fiber is collapsed. This was initiated by Witten [Wit85] and later rigorously treated by Bismut and Freed [BF86a] and independently by Cheeger [Che87]. Expanding on the earlier work of [BF86a, Che87, Wit85], Bismut and Cheeger [BC89], Mazzeo and Melrose [MM90] and then Dai [Dai91] studied the adiabatic limit for general fibrations of compact manifolds.

The eta invariant of compact quotients of $\operatorname{PSL}(2, \mathbb{R})$ was studied by Seade and Steer [SS87]. In this situation, the total space of the fibration is a circle bundle that fibers over a compact hyperbolic Riemann surface. There has been much interest in more general spectral problems for the case when the fibers are circles, for example, [AB98, Bec00, DZ95, Hit74, Nic98, Nic99, SS87, Zha94]. In all of these papers the base manifold is compact. When the base is not compact, there are very few papers on this subject except an unpublished paper of K. P. Wojciechowski [Woj] where he considered the adiabatic limit of the eta invariant over the base manifold $\mathbb{R}$.

Here we consider the spectral properties of the Dirac operator and the adiabatic limit of its eta invariant on a cofinite quotient $X$ in the case when the base Riemann surface has finite volume but is not compact,

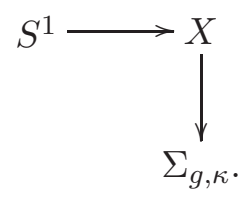

We assume that the base of the fibration is a complete hyperbolic Riemann surface $\Sigma_{g, \kappa}$ of genus $g$

Received 31 July 2007, accepted in final form 26 March 2008, published online 26 September 2008. 2000 Mathematics Subject Classification 58J28, 58J50 (primary), 11F72, 22E46 (secondary).

Keywords: eta invariant, adiabatic limit, fibered cusp operator, Selberg trace formula.

This journal is (C) Foundation Compositio Mathematica 2008. 


\section{P. LOYA, S. Moroianu And J. PARK}

with $\kappa$ cusps. One of our motivations is related to the index formula of a Dirac type operator over a locally symmetric space [Ste89] where one has to deal with the adiabatic limit of the eta invariants for the maximal faces, which have a fibration structure over a non-compact locally symmetric space. Ours is the simplest possible example of a fibration over a non-compact locally symmetric space, yet its spectral analysis turns out to be highly non-trivial.

We fix a spin structure and then replace the circle $S^{1}$ in (1.1) with a circle of radius $r$. We denote the corresponding Dirac operator by $D_{r}$. The first purpose of this paper is to study the spectral properties of $D_{r}$. In particular, we analyze the dependence of the continuous spectrum of $D_{r}$ on the choice of spin structure. Every end of $X$ is diffeomorphic to the trivial fibration with fiber $S^{1}$ over a corresponding end $\mathbb{R}^{+} \times S^{1}$ of $\Sigma_{g, \kappa}$. We say that the spin structure is trivial on an end of $X$ if it induces the trivial (that is, non-bounding) spin structure on the circle from the base.

Theorem 1.1. Let $X$ be a cofinite quotient of $\operatorname{PSL}(2, \mathbb{R})$. Fix a spin structure on $X$, and denote by $\kappa^{\mathrm{t}}$ the number of ends on which the induced spin structure is trivial. Then for all $r>0$, the continuous spectrum of the Dirac operator $D_{r}$ consists of $\kappa^{\mathrm{t}}$-copies of countably many families of half-lines

$$
\left(-\infty,-\frac{r}{2}-|m|\left(1+r^{-2}\right)^{1 / 2}\right] \cup\left[-\frac{r}{2}+|m|\left(1+r^{-2}\right)^{1 / 2},+\infty\right)
$$

indexed by odd integers $m \in 1+2 \mathbb{Z}$ if the spin structure along the $S^{1}$-fiber is trivial, or by even integers $m \in 2 \mathbb{Z}$ otherwise.

This theorem can be regarded as a generalization of the result of Bär in [Bar00] to the fibered cusp case where the continuous spectrum of $D_{r}$ depends on the spin structures of the $S^{1}$-fibers and of the $S^{1}$ cross sections of the base manifold $\Sigma_{g, \kappa}$ near the ends. Another novelty of this theorem is that the Riemannian metric over the cusps are not the exact fibered cusp metrics which have been extensively studied in, for example, [LM05, LMP07, Vai01]. It is because of the non-exactness of the fibered cusp metric that the continuous spectrum of $D_{r}$ is quite complicated.

The second main result of this paper is the computation of the adiabatic limit of the eta invariant of $D_{r}$ as the fiber is collapsed (that is, $r \rightarrow 0$ ). According to Theorem 1.1, the Dirac operator $D_{r}$ typically has non-empty continuous spectrum; moreover, the corresponding odd heat kernel of $D_{r}^{2}$ is not of trace class. Therefore, the standard definition of the eta function using the eigenvalues or the trace of the odd heat kernel is not valid unless the spin structure is non-trivial on every end. This requires us to define a regularized eta function, which is reminiscent of the $b$-eta function of Melrose [Mel93] and similar to the regularized eta function used by one of the present authors [Par05] over hyperbolic manifolds with cusps. Denote by $\eta\left(D_{r}, s\right)$ the eta function of $D_{r}$ defined through a regularized trace (see Definition in (4.6)); our main result is the following theorem.

TheOREM 1.2. We assume that the spin structure along the $S^{1}$-fiber is trivial.

(i) For a sufficiently small $r>0, \eta\left(D_{r}, s\right)$, defined for $\Re(s)>2$, extends meromorphically to $\mathbb{C}$ with a possible double pole at $s=1$ and possible simple poles at $\{2,0,-1,-2,-3, \ldots\}$. If the spin structure is non-trivial on each end, then $\eta\left(D_{r}, s\right)$ may have only simple poles at $\{2,1,0,-1,-2,-3, \ldots\}$.

(ii) Define $\eta\left(D_{r}\right)$ as the finite part at $s=0$ of the meromorphic extension $\eta\left(D_{r}, s\right)$. In the adiabatic limit, the following identity holds:

$$
\lim _{r \rightarrow 0} \eta\left(D_{r}\right)=-\frac{1}{12 \pi} \operatorname{Vol}\left(\Sigma_{g, \kappa}\right)=\frac{1}{6}(2-2 g-\kappa) .
$$

For the compact case and for the trivial spin structure, a result corresponding to the formula (1.2) in Theorem 1.2 was proved by Seade and Steer [SS87], who also obtained the value of the eta invariant 


\section{AdiabATiC LIMIT OF THE ETA INVARIANT}

of the original fibration (when $r=1$ ) by applying the Atiyah-Patodi-Singer index formula for a manifold with smooth boundary. However, in our non-compact case, it would be very difficult to prove an index formula for manifolds whose boundaries are manifolds with non-exact fibered cusp ends. Another possible approach (suggested by the referee) is to use the variation formula as in Bismut-Freed [BF86b]. This problem will be considered elsewhere.

This paper is organized as follows. In $\S 2$ we develop the required background material, including a discussion of spin structures and of the Dirac operator $D_{r}$. In $\S 3$ we analyze the Dirac operator in terms of the fibered cusp calculus of Mazzeo-Melrose [MM98] and we prove Theorem 1.1. In $\S 4$ we define the regularized eta invariant and in $\S \S 5,6$, and 7 we analyze the geometric and spectral sides of the Selberg trace formula in our context, which will be used to prove Theorem 1.2.

\section{Dirac operator and Spin structure}

In this section, we define the Dirac operator over a cofinite quotient of $\operatorname{PSL}(2, \mathbb{R})$ by a discrete subgroup. Equivalently, we consider the Lie group $G=\operatorname{SL}(2, \mathbb{R})$ and a discrete subgroup $\Gamma \subset G$ containing $\{ \pm 1\}$; then the quotient $\Gamma \backslash G$ is the same as the quotient $(\Gamma /\{ \pm 1\}) \backslash \operatorname{PSL}(2, \mathbb{R})$.

For $r \in(0, \infty)$ we define a family of metrics $g_{r}$ over $G$ such that the left translations of $E:=$ $r^{-1} C, A, H$ are orthonormal with respect to $g_{r}$ where $C, A, H$ is a basis of $\mathfrak{g}=\operatorname{sl}(2, \mathbb{R})$ given by

$$
C=\left(\begin{array}{cc}
0 & 1 \\
-1 & 0
\end{array}\right), \quad A=\left(\begin{array}{cc}
0 & 1 \\
1 & 0
\end{array}\right), \quad H=\left(\begin{array}{cc}
1 & 0 \\
0 & -1
\end{array}\right)
$$

Recall that the corresponding Levi-Civita connection $\nabla^{r}$ is determined by the Koszul formula

$$
\begin{aligned}
2 g_{r}\left(\nabla_{U}^{r} V, W\right)= & U g_{r}(V, W)+V g_{r}(W, U)-W g_{r}(U, V) \\
& +g_{r}([U, V], W)-g_{r}([U, W], V)-g_{r}([V, W], U)
\end{aligned}
$$

where $U, V, W$ denote vector fields over $G$.

Since $G$ is topologically the same as $S^{1} \times \mathcal{H}$ where $\mathcal{H}$ is the Poincaré upper half plane, there are two spin structures on $G$. We choose the one determined by the left invariant trivialization. Denoting the lifted connection to the spinor bundle by the same notation $\nabla^{r}$, we define the Dirac operator by

$$
\widehat{D}_{r}:=E \cdot \nabla_{E}^{r}+A \cdot \nabla_{A}^{r}+H \cdot \nabla_{H}^{r}
$$

where $U$. denotes the Clifford action by $U$. By a straightforward computation as in [Hit74, SS87], we obtain

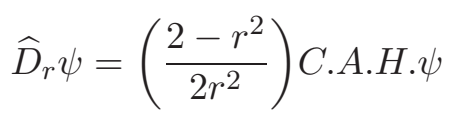

for a basic spinor $\psi$.

We twist $\widehat{D}_{r}$ by multiplying with the volume element $\omega:=E . A . H$. to define $\widetilde{D}_{r}$, that is,

$$
\widetilde{D}_{r}:=E \cdot A \cdot H \cdot \widehat{D}_{r},
$$

which has the following simplified form,

$$
\widetilde{D}_{r} \psi=\left(\frac{2-r^{2}}{2 r}\right) \psi
$$

for a basic spinor $\psi$. The Clifford algebra generated by $E, A, H$ has the Pauli matrix representation given by

$$
E \mapsto\left(\begin{array}{cc}
i & 0 \\
0 & -i
\end{array}\right), \quad A \mapsto\left(\begin{array}{cc}
0 & 1 \\
-1 & 0
\end{array}\right), \quad H \mapsto\left(\begin{array}{cc}
0 & -i \\
-i & 0
\end{array}\right)
$$


Then we have

$$
\omega E \mapsto-i\left(\begin{array}{cc}
1 & 0 \\
0 & -1
\end{array}\right), \quad \omega A \mapsto\left(\begin{array}{cc}
0 & 1 \\
-1 & 0
\end{array}\right), \quad \omega H \mapsto i\left(\begin{array}{cc}
0 & 1 \\
1 & 0
\end{array}\right) .
$$

It follows that for any spinor $\alpha \psi_{1}+\beta \psi_{2}$ written in terms of basic spinors $\psi_{1}, \psi_{2}$ and smooth functions $\alpha, \beta$ on $G$, we have the following representation of $\widetilde{D}_{r}$,

$$
\widetilde{D}_{r}\left(\begin{array}{c}
\alpha \\
\beta
\end{array}\right)=\left(\frac{2-r^{2}}{2 r}\right)\left(\begin{array}{l}
\alpha \\
\beta
\end{array}\right)+\left(\begin{array}{cc}
-i E & A+i H \\
-A+i H & i E
\end{array}\right)\left(\begin{array}{l}
\alpha \\
\beta
\end{array}\right) .
$$

Now we let

$$
Z:=-i C, \quad 2 X_{+}:=A-i H, \quad 2 X_{-}:=A+i H .
$$

(Our convention is slightly different from that in [SS87].) These vector fields satisfy

$$
\left[Z, X_{+}\right]=2 X_{+}, \quad\left[Z, X_{-}\right]=-2 X_{-}, \quad\left[X_{+}, X_{-}\right]=Z,
$$

and we have

$$
\widetilde{D}_{r}=\left(\frac{2-r^{2}}{2 r}\right)+\left(\begin{array}{cc}
r^{-1} Z & 2 X_{-} \\
-2 X_{+} & -r^{-1} Z
\end{array}\right) \quad \text { acting on } C^{\infty}(G) \oplus C^{\infty}(G) .
$$

It is also easy to check that

$$
\widetilde{D}_{r}^{2}=\left(\begin{array}{cc}
-\left(A^{2}+H^{2}+r^{-2} C^{2}\right) & 0 \\
0 & -\left(A^{2}+H^{2}+r^{-2} C^{2}\right)
\end{array}\right)+\text { lower order terms, }
$$

hence the Dirac Laplacian $\widetilde{D}_{r}^{2}$ is a generalized Laplacian whose principal symbol is given by the metric $g_{r}$, as expected.

To define the Dirac operator over $X=\Gamma \backslash G$, let us discuss the spin structures on $X=\Gamma \backslash G$, which will play a crucial role throughout this paper. First recall that there are $\left|H^{1}\left(X, \mathbb{Z}_{2}\right)\right|$-number of spin structures over $X$, since every orientable three-dimensional manifold is spin. This can be understood from the following diagram,

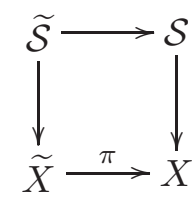

where $\widetilde{\mathcal{S}}, \mathcal{S}$ are $\operatorname{Spin}(3)$ bundles over the universal cover $\widetilde{X}$ and over $X$, respectively. Since $\widetilde{\mathcal{S}} \cong$ $\widetilde{X} \times \operatorname{Spin}(3)$ and $\widetilde{\mathcal{S}} \cong \pi^{*} \mathcal{S}$, the possible Spin bundle $\mathcal{S}$ is given by a $\mathbb{Z}_{2}$-representation $\rho$ of $\pi_{1}(X)$ as follows:

$$
\mathcal{S}_{\rho}=\tilde{X} \times{ }_{\rho} \operatorname{Spin}(3)
$$

with the obvious $\mathbb{Z}_{2}$-action on $\operatorname{Spin}(3)$. Therefore, each $\mathbb{Z}_{2}$-representation of $\pi_{1}(X)$ provides us with an inequivalent spin structure on $X$. Recall that

$$
\pi_{1}(X)=\left\{x_{i}, y_{i}, h_{j}, k \mid 1 \leqslant i \leqslant g, 1 \leqslant j \leqslant \kappa, \prod_{i=1}^{g}\left[x_{i}, y_{i}\right] \prod_{j=1}^{\kappa} h_{j}=1,\left[x_{i}, k\right]=\left[y_{i}, k\right]=\left[h_{j}, k\right]=1\right\},
$$

where $g, \kappa$ denote the genus and the number of cusps, respectively, of the base Riemann surface $\Sigma_{g, \kappa}$ of the fibration (1.1).

Among spin structures, there are spin structures which are determined by those $\mathbb{Z}_{2}$-representations $\rho$ of $\pi_{1}(X)$ with $\rho\left(h_{j}\right)=-1$ for some $j$. In [Bar00] such a spin structure over the Riemann surface $\Sigma_{g, \kappa}$ is called non-trivial along the cusp corresponding to $j$. Following [Bar00], we call such a spin structure non-trivial along the cusp if $\rho\left(h_{j}\right)=-1$ for the corresponding $j$, and a totally 


\section{AdiABATIC LIMIT OF THE ETA INVARIANT}

non-trivial spin structure if it is determined by a $\mathbb{Z}_{2}$-representation $\rho$ with

$$
\rho\left(h_{j}\right)=-1 \quad \text { for all } j=1, \ldots, \kappa .
$$

From the relations of the generators of $\pi_{1}(X)$, there exists an obstruction for this:

$$
\prod_{j=1}^{\kappa} \rho\left(h_{j}\right)=1 .
$$

Hence, for a spin structure to be totally non-trivial the number of cusps $\kappa$ should be even. We also distinguish two classes of spin structures according to the (non-)triviality of the spin structure along the fiber $S^{1} /\{ \pm 1\}$. We call the spin structure trivial along the fiber if the spin structure is trivial along the fiber $S^{1} /\{ \pm 1\}$ (or, equivalently, if the representation $\rho$ maps the generator $k$ to 1 ), and non-trivial along the fiber otherwise. Note that if the spin structure is trivial along the $S^{1} /\{ \pm 1\}$ fiber, this spin structure does not extend to a spin structure over the disc bundle over $\Sigma_{g, \kappa}$. From the above discussion we have the following result.

Proposition 2.1. There are $2^{2 g+\kappa}$ spin structures over $X=\Gamma \backslash G$. There exist totally non-trivial spin structures over $X$ if and only if $\kappa$ is even.

For the trivial representation of $\pi_{1}(X)$, the resulting Spin bundle denoted by $\mathcal{S}_{1}$ is topologically trivial, and is determined by the left invariant trivialization over $X=\Gamma \backslash G$. The associated spinor bundle $\Sigma_{1}=\mathcal{S}_{1} \times_{\operatorname{Spin}(3)} \Sigma(3)$ (where $\Sigma(3) \cong \mathbb{C}^{2}$ is the spinor representation of $\left.\operatorname{Spin}(3) \cong \mathrm{SU}(2)\right)$ is therefore also trivial and if $\Sigma_{\rho}$ denotes the spinor bundle associated to a representation $\rho$, that is, $\Sigma_{\rho}=\mathcal{S}_{\rho} \times_{\operatorname{Spin}(3)} \Sigma(3)$, then

$$
\Sigma_{\rho}=\Sigma_{1} \otimes \mathbb{C}_{\rho}
$$

where $\mathbb{C}_{\rho} \rightarrow X$ is the flat line bundle associated to $\rho$.

If $D_{r}$ denotes the induced Dirac operator from $\widetilde{D}_{r}$ pushed down to $X$, then from the definition of $\widetilde{D}_{r}$ over $G$ and the equality $(2.4)$, we can see that

$$
D_{r}=\left(\frac{2-r^{2}}{2 r}\right)+\left(\begin{array}{cc}
r^{-1} Z & 2 X_{-} \\
-2 X_{+} & -r^{-1} Z
\end{array}\right) \quad \text { acting on } C_{0}^{\infty}(\Gamma \backslash G, \chi),
$$

where $C_{0}^{\infty}(\Gamma \backslash G, \chi)(\chi=\rho \oplus \rho)$ consists of the smooth functions with co-compact supports such that $f(\gamma x)=\chi(\gamma) f(x)$ for $\gamma \in \pi_{1}(X), x \in G$. We also denote the $L^{2}$-closure of $D_{r}$ (with respect to a certain metric explained in (4.1)) by $D_{r}$, that is,

$$
D_{r}: L^{2}(\Gamma \backslash G, \chi) \longrightarrow L^{2}(\Gamma \backslash G, \chi) .
$$

\section{Analysis of fibered cusp operators}

In this section we show that the metrics $g_{r}$ are of conformal fibered cusp type. Consequently, we show that the Dirac operators $D_{r}$ belong to the class of weighted fibered cusp operators introduced by Mazzeo and Melrose [MM98], and we prove Theorem 1.1.

First we introduce some subgroups of $G=\mathrm{SL}(2, \mathbb{R})$,

$$
N_{0}=\left\{\left(\begin{array}{ll}
1 & x \\
0 & 1
\end{array}\right)\right\}, \quad A_{0}=\left\{\left(\begin{array}{cc}
e^{u / 2} & 0 \\
0 & e^{-u / 2}
\end{array}\right)\right\}, \quad K=\left\{\left(\begin{array}{cc}
\cos \theta & \sin \theta \\
-\sin \theta & \cos \theta
\end{array}\right)\right\}
$$

where $x, u, \theta \in \mathbb{R}$. Then the standard parabolic subgroup $P_{0}$ is given by $N_{0} A_{0} Z$ where $Z=\{ \pm 1\} \subset$ $K$ and any parabolic subgroup $P$ of $G$ is conjugate to $P_{0}$ by an element $k_{P}$ in $K$. A parabolic subgroup $P$ has a decomposition $P=N_{P} A Z$ where $N_{P}$ is the derived group of $P$ and $A$ is any conjugate of $A_{0}$ in $P$, to be called a Cartan subgroup. It is clear that $A_{0}$ is the unique Cartan 


\section{P. Loya, S. Moroianu And J. PARK}

subgroup $P_{0}$ with Lie algebra orthogonal to that of $K$. Therefore, $P$ has a unique Cartan subgroup with the same property. From now on, we assume that the pair $(P, A)$ satisfies this condition. For such a pair $(P, A)$ with $N=N_{P}$, we have the Iwasawa decomposition $G=N A K$.

For a given $\Gamma \subset G$, a parabolic subgroup $P$ of $G$ is called $\Gamma$-cuspidal if $N=N_{P}$ contains a nontrivial element of $\Gamma$. It is well known that the finitely many ends of $X=\Gamma \backslash G$ are parametrized by $\Gamma$-conjugacy classes $\{P\}_{\Gamma}=\left\{\gamma P \gamma^{-1} \mid \gamma \in \Gamma / \Gamma_{P}\right\}$ where $\Gamma_{P}:=\Gamma \cap P$. We refer to [Bor97, ch. 3] for an explanation concerning this fact. Let $P$ be a $\Gamma$-cuspidal parabolic subgroup of $G$ corresponding to one end of $X=\Gamma \backslash G$. This subgroup determines a cusp $c_{P}$, an incomplete manifold which is identified with a neighborhood of the cuspidal end of the quotient $\Gamma_{P} \backslash G$.

Assume first that $P=P_{0}$ is the standard parabolic subgroup of $G$. The manifold $\Gamma_{P} \backslash G$ has two commuting free $S^{1}$ actions: the action of $K$ to the right and that of $\Gamma_{N_{0}} \backslash N_{0}$ to the left where $\Gamma_{N_{0}}:=\Gamma \cap N_{0}$. The first $S^{1}$ action, in fact, exists globally on $X=\Gamma \backslash G$, while the second one exists only on the cusp. Let $\gamma_{l}:=\left(\begin{array}{ll}1 & l \\ 0 & 1\end{array}\right)$ be the generator of $\Gamma_{N_{0}}$. Then $\Gamma_{P} \backslash G$ is identified with $\mathbb{R} / l \mathbb{Z} \times \mathbb{R} \times \mathbb{R} / 2 \pi \mathbb{R}$ by the map

$$
(x, u, \theta) \mapsto\left(\begin{array}{cc}
1 & x \\
0 & 1
\end{array}\right)\left(\begin{array}{cc}
e^{u / 2} & 0 \\
0 & e^{-u / 2}
\end{array}\right)\left(\begin{array}{cc}
\cos \theta & \sin \theta \\
-\sin \theta & \cos \theta
\end{array}\right) .
$$

By projection on the last two terms, we view this as the total space of a fibration with fiber $S^{1}$. Note that this fibration makes sense only near the end and is not the fibration in (1.1) where the roles of the two $S^{1}$ are reversed.

As seen above, the spinor bundle corresponding to the representation $\rho$ is the spinor bundle for the trivial representation, twisted by the flat line bundle $\mathbb{C}_{\rho}$ defined by $\rho$. The Dirac operator on $G$ has been computed in (2.6) with respect to the orthonormal vector fields $E=r^{-1} C, A, H$ defined in (2.1) and the representation $\chi$. The same expression holds on the spinor bundle on the cusp $\Gamma_{P} \backslash G$, where the vector fields $E, A, H$ now act on $\Sigma_{\rho}$. There is no ambiguity about the action of these vector fields since the twisting bundle $\mathbb{C}_{\rho}$ is flat.

Introduce the function $\nu:=e^{-u}$ on the cusp and glue the 'boundary at infinity' $\mathbb{R} / l \mathbb{Z} \times\{\nu=$ $0\} \times \mathbb{R} / 2 \pi \mathbb{R}$ to the cusp, thus obtaining a manifold with boundary $\overline{\Gamma_{P} \backslash G}$. The $S^{1}$-fibration structures extend to the boundary. We show that for each fixed $r$, the metric $g_{r}$ on $X$ is conformal to a fibered cusp metric (with respect to the fibration of the boundary induced from the $\Gamma_{N_{0}} \backslash N_{0}$ action). In the coordinates $\left(x, \nu=e^{-u}, \theta\right)$ of $\Gamma_{P} \backslash G$, the coefficients of a matrix $\left(\begin{array}{ll}a & b \\ c & d\end{array}\right)$ are given by the inverse of the map (3.2):

$$
x=\frac{a c+b d}{c^{2}+d^{2}}, \quad \nu=c^{2}+d^{2}, \quad \theta=-\arctan \left(\frac{c}{d}\right) .
$$

We then compute

$$
\begin{aligned}
& E=r^{-1} \partial_{\theta}, \\
& A=-\cos 2 \theta \partial_{\theta}+2 \nu^{-1} \cos 2 \theta \partial_{x}-2 \nu \sin 2 \theta \partial_{\nu}, \\
& H=\sin 2 \theta \partial_{\theta}-2 \nu^{-1} \sin 2 \theta \partial_{x}-2 \nu \cos 2 \theta \partial_{\nu} .
\end{aligned}
$$

These equalities also can be found in [GGP69, p. 52] or [Lan75, p. 115]. It follows that in the coordinates $(x, \nu, \theta)$ the metric $g_{r}$ is given by

$$
\frac{1}{4 \nu^{2}} d \nu^{2}+\frac{\nu^{2}}{4} d x^{2}+r^{2}\left(d \theta+\frac{\nu}{2} d x\right)^{2},
$$

thus

$$
g_{\Phi}:=\frac{4}{\nu^{2}} g_{r}=\left(\frac{d \nu}{\nu^{2}}\right)^{2}+r^{2}\left(\frac{2 d \theta}{\nu}+d x\right)^{2}+d x^{2} .
$$




\section{AdiABATIC LIMIT OF THE ETA INVARIANT}

This is what is called a fibered cusp metric, or a smooth metric on the fibered cusp tangent bundle. To define this bundle, consider the subalgebra ${ }^{\Phi} \mathcal{V}$ of the Lie algebra $\mathcal{V}$ of smooth vector fields on the manifold with boundary $\overline{\Gamma_{P} \backslash G}$, spanned over $C^{\infty}\left(\overline{\Gamma_{P} \backslash G}\right)$ by the vector fields

$$
V_{\nu}:=\nu^{2} \partial_{\nu}, \quad V_{\theta}:=\nu \partial_{\theta}, \quad V_{x}:=\partial_{x} .
$$

This sub-algebra is by definition a free $C^{\infty}\left(\overline{\Gamma_{P} \backslash G}\right)$-module so it is the space of sections of a smooth vector bundle over $C^{\infty}\left(\overline{\Gamma_{P} \backslash G}\right)$. This vector bundle is denoted by ${ }^{\Phi} T \Gamma_{P} \backslash G$ and it comes equipped with a bundle morphism to the usual tangent bundle $T \overline{\Gamma_{P} \backslash G}$, induced from the inclusion of the spaces of sections ${ }^{\Phi} \mathcal{V} \hookrightarrow \mathcal{V}$, which is an isomorphism over $\Gamma_{P} \backslash G$.

Since ${ }^{\Phi} \mathcal{V}$ is a Lie algebra and the metric $g_{\Phi}$ defined above is non-degenerate and smooth on fibered cusp vector fields, it follows immediately from the Cartan formula that the Levi-Civita connection on $\Gamma_{P} \backslash G$ with respect to the metric $g_{\Phi}$ extends to the boundary in the sense that for every $V_{i}, V_{j}, V_{k} \in{ }^{\Phi} \mathcal{V}$, we have

$$
\left\langle\nabla_{V_{i}} V_{j}, V_{k}\right\rangle \in C^{\infty}\left(\overline{\Gamma_{P} \backslash G}\right) .
$$

The spinor bundle $\Sigma_{\rho}$ extends over the boundary, such that the Clifford multiplication by $V_{i}$ is a smooth map. Now take the orthonormal frame

$$
V_{1}:=V_{\nu}, \quad V_{2}:=V_{x}-V_{\theta} / 2, \quad V_{3}:=\frac{1}{2 r} V_{\theta}
$$

Its relationship to the global frame $(E, A, H)$ is deduced from (3.3):

$$
\begin{aligned}
V_{1} & =-\frac{\nu}{2}(\sin 2 \theta A+\cos 2 \theta H), \\
V_{2} & =\frac{\nu}{2}(\cos 2 \theta A-\sin 2 \theta H), \\
V_{3} & =\frac{\nu}{2} E .
\end{aligned}
$$

Denote by $V$ a local lift to the spinor bundle of the orthonormal frame $\left(V_{1}, V_{3}, V_{2}\right)$. Let $\sigma$ : $\overline{\Gamma_{P} \backslash G} \rightarrow \Sigma(3)$ be a smooth map into the 3 -spinor representation space. It follows from the local formula

$$
\begin{aligned}
D_{\Phi}[V, \sigma] & =\sum_{i=1}^{3} c\left(V_{i}\right)\left(\left[V, V_{i}(\sigma)\right]+\frac{1}{2} \sum_{j<k} c\left(V_{j}\right) c\left(V_{k}\right)\left\langle\nabla_{V_{i}} V_{j}, V_{k}\right\rangle[V, \sigma]\right) \\
& =\left(c\left(V_{1}\right)\left(\nu^{2} \partial_{\nu}-\frac{\nu}{2}\right)+c\left(V_{2}\right) \partial_{\theta} \frac{\nu}{2 r}+c\left(V_{3}\right)\left(\partial_{x}-\frac{\nu \partial_{\theta}}{2}\right)+r \frac{\nu}{4}\right)[V, \sigma]
\end{aligned}
$$

that the Dirac operator with respect to $g_{\Phi}$ (defined first on compactly supported spinors over $\Gamma_{P} \backslash G$ ) extends to smooth spinors up to the boundary. Such an operator, a combination of fibered cusp vector fields and of smooth bundle endomorphisms down to the boundary $\{\nu=0\}$, is called a fibered cusp differential operator. Thus,

$$
D_{\Phi} \in \operatorname{Diff}_{\Phi}^{1}\left(\overline{\Gamma_{P} \backslash G}, \Sigma_{\rho}\right) .
$$

The Dirac operator changes very nicely with respect to conformal changes of the metric. We simply have

$$
D_{r}=2 \nu^{-2} \circ D_{\Phi} \circ \nu
$$

so for $r>0$, the Dirac operator $D_{r}$ is a differential operator in $\nu^{-1} \operatorname{Diff}_{\Phi}^{1}\left(\overline{\Gamma_{P} \backslash G}, \Sigma_{\rho}\right)$.

The normal operator $\mathcal{N}\left(D_{\Phi}\right)(\theta, \xi, \tau)$ of $D_{\Phi}$ (see [MM98]) is obtained by formally replacing

$$
V_{\nu} \mapsto i \xi, \quad V_{\theta} \mapsto i \tau
$$

and then restricting to $\nu=0$. The result is a family of differential operators on the fibers of the boundary fibration (the $x$-circles) with coefficients in the spinor bundle, with parameters $\theta \in S^{1}$, 
$(\xi, \tau) \in \mathbb{R}^{2}:$

$$
\mathcal{N}\left(D_{\Phi}\right)(\theta, \xi, \tau)=c\left(V_{1}\right) i \xi+c\left(V_{2}\right)\left(\partial_{x}-\frac{i \tau}{2}\right)+c\left(V_{3}\right) \frac{i \tau}{2 r}
$$

Definition 3.1. The operator $D_{\Phi}$ is called fully elliptic if $\mathcal{N}\left(D_{\Phi}\right)(\theta, \xi, \tau)$ is invertible for all $(\theta, \xi, \tau) \in S^{1} \times \mathbb{R}^{2}$.

If $D_{\Phi}$ is fully elliptic, then by the results of [MM98] it has a parametrix inside the calculus of fibered cusp pseudodifferential operators $\Psi_{\Phi}^{-1}(X)$ modulo compact operators.

Proof of Theorem 1.1. According to the decomposition principle (see, for example, [Bar00, Proposition 1]), the essential spectrum of $D_{r}$ can be computed outside a compact subset of $X$, thus it is a superposition of the essential spectra of any self-adjoint extension of $D_{r}$ over each cuspidal end $c_{P}$ defined by $\nu_{P}<\epsilon_{P}$. We must make sure that such an extension exists (the Dirac operator on a manifold with boundary may not admit self-adjoint extensions, for example, on $\mathbb{R}^{+}$). We may take, for instance, the Atiyah-Patodi-Singer boundary condition at the torus boundary $\left\{\nu_{P}=\epsilon_{P}\right\}$. Special care is needed for the nullspace of the Dirac operator along the torus, we only allow harmonic spinors of the form $\left(u, c\left(V_{3}\right) u\right)$ in the domain where $u$ is in the $i$-eigenspace of $c\left(V_{1}\right)$.

Since any $\Gamma$-parabolic subgroup $P$ is conjugated by an element in the maximal compact subgroup $K$ to the standard parabolic subgroup $P_{0}$, we see that the cusp corresponding to $P$ is isometric to the 'canonical' cusp $P_{0} \backslash G$. Thus, without loss of generality we work with the canonical parabolic subgroup $P_{0}$.

We have seen above that $D_{r}$ belongs to $\nu^{-1} \operatorname{Diff}_{\Phi}^{1}\left(\overline{\Gamma_{P} \backslash G}, \Sigma_{\rho}\right)$ near the cuspidal end.

Lemma 3.2. The fibered cusp operator $D_{\Phi}$ is fully elliptic on the cusp $c_{P}$ if and only if the spin structure is non-trivial along $c_{P}$.

Proof. We have computed above the normal operator $\mathcal{N}:=\mathcal{N}\left(D_{\Phi}\right)(\theta, \xi, \tau)$. Clearly, $\mathcal{N}$ is an elliptic self-adjoint operator on the circle in the variable $x$. Therefore, $\mathcal{N}$ is invertible if and only if $\mathcal{N}^{2}$ is. Now by the anti-commutation of the Clifford variables,

$$
\mathcal{N}^{2}=\xi^{2}+\frac{\tau^{2}}{4 r^{2}}+\left(i \partial_{x}-\frac{\tau}{2}\right)^{2} .
$$

This family of operators is independent of $\theta$; it is strictly positive (hence, invertible) for $(\xi, \tau) \neq$ $0 \in \mathbb{R}^{2}$. For $\xi=\tau=0, \mathcal{N}=-\partial_{x}^{2}$, so $\operatorname{ker}(\mathcal{N})$ is made of those spinors which are constant in $x$ in the trivialization $V$ of the spinor bundle. For fixed $\theta$, such spinors exist globally on the $x$-circle if and only if the local lift $V$ satisfies $V_{x=l}=V_{x=0}$. Now the frame $\left(V_{1}, V_{2}, V_{3}\right)$ is obtained from $(E, A, H)$ by the transformation (3.4) which is constant in $x$; thus, the lift $V$ exists globally around the cusp if and only if the lift of $(E, A, H)$ exists globally around the cusp, which is by definition equivalent to the triviality of the spin structure around the cusp $c_{P}$.

If $D_{\Phi}$ is fully elliptic, it follows from the above lemma and from the general theory of fibered cups operators that $D_{r}=2 \nu^{-2} D_{\Phi} \nu$ has a parametrix $Q \in \nu \Psi_{\Phi}^{-1}$ over the cusp $c_{P}$, modulo compact operators. However, $Q$ itself is compact due to the decaying weight $\nu$; hence, the self-adjoint operator $\mathcal{D}_{r}$ has pure-point spectrum over the cusp.

Conversely, assume that the spin structure is trivial along the cusp. The operator $D_{r}=2 \nu^{-2} D_{\Phi} \nu$ computed in (3.5) has constant coefficients in $x$, thus it preserves the orthogonal decomposition into zero modes and high-energy modes

$$
L^{2}\left(\Gamma_{P} \backslash G \cap\left\{\nu_{P}<\epsilon_{P}\right\}, \Sigma_{\rho}\right)=: \mathcal{H}_{0} \oplus \mathcal{H}^{\prime},
$$




\section{AdiABATIC LIMIT OF THE ETA INVARIANT}

where $\mathcal{H}_{0}$ is the space of spinors constant in $x$ in the trivialization $V$ (we have seen above that $V$ exists globally around the cusp if the spin structure is trivial along $c_{P}$ ) and $\mathcal{H}^{\prime}$ its orthogonal complement. Over $\mathcal{H}^{\prime}$, by the same argument as above, there exists a compact parametrix of $D_{r}$ inside the fibered cusp calculus. Thus, the essential spectrum of $D_{r}$ over the cusp $c_{P}$ only arises from the zero modes, that is, it is the essential spectrum of the operator

$$
2 \nu^{-1}\left(c\left(V_{1}\right)\left(\nu \partial_{\nu}-\frac{1}{2}\right)-c\left(V_{2}\right) \frac{\partial_{\theta}}{2}+c\left(V_{3}\right) \frac{\partial_{\theta}}{2 r}-\frac{r}{4}\right) \nu
$$

acting in $L^{2}\left([0, \epsilon) \times S^{1}, \Sigma_{\rho}, d \nu d \theta\right)$ with any boundary condition at $\epsilon$ which makes it self-adjoint. We conjugate this operator through the Hilbert space isometry

$$
L^{2}(d \nu d \theta) \rightarrow L^{2}\left(\frac{d \nu}{\nu} d \theta\right), \quad \phi \mapsto \nu^{\frac{1}{2}} \phi
$$

We obtain the operator

$$
A_{r}=2 c\left(V_{1}\right) \nu \partial_{\nu}+\left(\frac{c\left(V_{3}\right)}{r}-c\left(V_{2}\right)\right) \partial_{\theta}-\frac{r}{2}
$$

This can be again decomposed according to the frequencies in the $\theta$ variable. Note that although the local lift $V$ may not exist globally, the ambiguity is locally constant so that the operator $i \partial_{\theta}$ is well defined; moreover, it clearly commutes with $A_{r}$.

From (3.4), the frame $\left(V_{1}, V_{2}, V_{3}\right)$ is obtained (after rescaling) from the frame $(E, A, H)$ by a complete rotation around the $E$ axis in time $\pi$. Such a rotation is a generator of $\pi_{1}(\mathrm{SO}(3))=\mathbb{Z} / 2 \mathbb{Z}$. Hence, $V$ exists globally around the $\theta$ circle if and only if the lift of $(E, A, H)$ does not, that is, if the spin structure is non-trivial along the fiber $S^{1} /\{ \pm 1\}$. Otherwise, if the spin structure is trivial along the fiber $S^{1} /\{ \pm 1\}$, then after time $\pi$ the lift $V$ changes sign.

A spinor $[V, \sigma]$ is in the $m$-eigenspace of $i \partial_{\theta}$ if and only if

$$
\sigma(t+\theta)=e^{-i m \theta} \sigma(t) .
$$

The resulting spinor should be $\pi$-periodic (since we work on $\operatorname{PSL}(2, \mathbb{R}$ ), we have assumed that $-1 \in \Gamma)$. We distinguish between two cases.

(i) The spin structure is non-trivial along the $S^{1} /\{ \pm 1\}$ fiber. Then $V(\pi)=V(0)$ so we want $\sigma(\pi)=\sigma(0)$. The eigenspinor equation (3.6) gives $m \in 2 \mathbb{Z}$.

(ii) The spin structure is trivial along the $S^{1} /\{ \pm 1\}$ fiber. Then $V(\pi)=-V(0)$ so we want $\sigma(\pi)=$ $-\sigma(0)$. The eigenspinor equation (3.6) gives $m \in 1+2 \mathbb{Z}$.

In both cases, the $m$-eigenspaces are two-dimensional representation spaces for $c\left(V_{j}\right), j=1,2,3$.

Denote by $A_{r, m}$ the action of $A_{r}$ on the $m$-eigenspace of $i \partial_{\theta}$. We obtain a $b$-operator $A_{r, m}$ (in the sense of Melrose) in $L^{2}\left([0, \epsilon), \mathbb{C}^{2}, \nu^{-1} d \nu\right)$

$$
A_{r, m}=2 c\left(V_{1}\right) \nu \partial_{\nu}-c\left(\frac{V_{3}}{r}-V_{2}\right) i m-\frac{r}{2} .
$$

The $b$-normal operator of $A_{r, m}$ is obtained by replacing $\nu \partial_{\nu}$ with $i s$ where $s$ is a complex parameter. One knows from the general theory of $b$-operators [Mel93] that the following statements are equivalent:

- $\lambda$ does not belong to the essential spectrum of $A_{r, m}$, that is, $A_{r, m}-\lambda$ is Fredholm;

- $\mathcal{N}\left(A_{r, m}\right)(s)-\lambda$ is invertible for all $s \in \mathbb{R}$.

We use now the representation

$$
c\left(V_{1}\right)=\left[\begin{array}{cc}
i & 0 \\
0 & -i
\end{array}\right], \quad c\left(\frac{V_{3}}{r}-V_{2}\right)=\left[\begin{array}{cc}
0 & 1+r^{-2} \\
-1 & 0
\end{array}\right]
$$


so that

$$
\mathcal{N}\left(A_{r, k}\right)(s)=\left[\begin{array}{cc}
-2 s & -i m\left(1+r^{-2}\right) \\
i m & 2 s
\end{array}\right]-\frac{r}{2} .
$$

An easy computation shows that $\mathcal{N}\left(A_{r, k}\right)(s)-\lambda$ is invertible for all $s \in \mathbb{R}$ exactly for

$$
\lambda \in\left(-\frac{r}{2}-|m|\left(1+r^{2}\right)^{\frac{1}{2}} r^{-1},-\frac{r}{2}+|m|\left(1+r^{2}\right)^{\frac{1}{2}} r^{-1}\right) .
$$

Thus, the essential spectrum of $D_{r}$ is the superposition of the complements of these intervals for each $k$ and for each cusp $c_{P}$ with trivial spin structure.

\section{Regularized trace and Geometric side}

In this section, we study the relation of certain regularized trace of the odd heat operator of $D_{r}$ with the geometric side of the Selberg trace formula.

To use harmonic analysis over $G$, we need to fix the Haar measures over $G$ and its subgroups. First, the parametrizations in (3.1) for $A_{0}, N_{0}$ carry the Lebesgue measure $d u, d n$ from $\mathbb{R}$ to $A_{0}, N_{0}$. Now we fix Haar measures on $K$ by $\operatorname{vol}(K / Z)=1$ and on $G$ by

$$
\int_{G} f(g) d g=\int_{N_{0}} \int_{A_{0}} \int_{K} f\left(n a_{u} k\right) e^{-u} d n d u d k
$$

for $f_{0} \in C_{0}(G)$ and $a_{u}=\operatorname{diag}\left(e^{u / 2}, e^{-u / 2}\right)$. For $a_{P, u}:=k_{P}^{-1} a_{u} k_{P} \in A=k_{P}^{-1} A_{0} k_{P}$, we put

$$
H_{P}(g)=u \quad \text { for } g \in N a_{P, u} K \text {. }
$$

The Iwasawa decomposition $\mathcal{H} \cong G / K \cong N A$ provides a parametrization of the geodesics $n A \cdot i \subset \mathcal{H}$ to infinity. The parameter value is given by the function $H_{P}$ whose potential curves are $N$-orbits (horocycles) on $\mathcal{H}$. However, this parametrization is not adapted to $\Gamma$. To rectify this, we replace $k_{P}$ by $g_{P}=a_{u_{P}} k_{P}$ where $e^{-u_{P}}=\operatorname{vol}\left(\Gamma_{N} \backslash N\right)$ where $\Gamma_{N}:=\Gamma \cap N$. For the new parameter

$$
H_{P}(g)+u_{P}=H_{P_{0}}\left(g_{P} g\right),
$$

then the value 0 of this new parameter corresponds to the horocycle whose projection on $\Gamma \backslash \mathcal{H}$ has length 1 .

For $\phi \in \mathrm{H}:=L^{2}(Z \backslash K)=\left\langle e^{i m \theta} \mid m \in 2 \mathbb{Z}\right\rangle$ and $s \in \mathbb{C}$, we extend $\phi$ to $G$ by

$$
\phi_{s}\left(n a_{u} k\right)=e^{s u} \phi(k) \text { for } n \in N_{0}, k \in K \text {. }
$$

These functions constitute the Hilbert space $\mathrm{H}_{s} \cong \mathrm{H}$ in which the representation $\pi_{s}$ induced from the parabolic subgroup $P_{0}=N_{0} A_{0} Z$ acts as

$$
\left(\pi_{s}(g) \phi_{s}\right)(x)=\phi_{s}(x g) .
$$

From now on, we assume that $\mathfrak{P}=\left\{P_{1}, \ldots, P_{\kappa}\right\}$ is a set of representatives for $\Gamma$-conjugacy classes of the cuspidal parabolic subgroups and that the spin structure over $c_{P_{i}}$ for $1 \leqslant i \leqslant \kappa^{\mathrm{t}}$ is trivial. We also assume that the representation $\rho$ maps the generator $k \in \pi_{1}(X)$ to the identity 1 , thus we consider only spin structures which are trivial along the $S^{1} /\{ \pm 1\}$-fiber. For the representation space $V \cong \mathbb{C}^{2}$ of $\chi=\rho \oplus \rho$, we let $V^{P}$ be the invariant subspace of $V$ under the action $\left.\chi\right|_{\Gamma_{P}}$. Then

$$
V^{P_{i}}= \begin{cases}V & \text { if } 1 \leqslant i \leqslant \kappa^{\mathrm{t}}, \\ \{0\} & \text { if } \kappa^{\mathrm{t}}+1 \leqslant i \leqslant \kappa .\end{cases}
$$

For a cuspidal parabolic subgroup $P, s \in \mathbb{C}$ with $\Re(s)>1$ and $\phi \in \mathbf{H} \otimes V^{P}$, the Eisenstein series 
$E(P, \phi, s)$ is defined by

$$
E(P, \phi, s)(g):=\sum_{\gamma \in \Gamma / \Gamma_{P}} \chi(\gamma) \phi_{s}\left(g_{P} \gamma^{-1} g\right)
$$

We refer to $[$ Hof $94, \S 5]$ for more about the Eisenstein series where the Hecke operator action is also involved. Note that there is no Eisenstein series attached to $P_{i}$ if $\kappa^{\mathrm{t}}+1 \leqslant i \leqslant \kappa$. The Eisenstein series $E(P, \phi, s)$ converges absolutely and locally uniformly for $\Re(s)>1$ and has the meromorphic extension over $\mathbb{C}$. In particular, $E(P, \phi, s)$ is an automorphic form, that is,

$$
E(P, \phi, s)(\gamma g)=\chi(\gamma) E(P, \phi, s)(g) \quad \text { for } \gamma \in \Gamma, g \in G .
$$

For $\phi \in \mathrm{H} \otimes V^{\text {cst }}$ with $V^{\text {cst }}:=\bigoplus_{P \in \mathfrak{P}} V^{P}$, we define

$$
E(\phi, s)=\sum_{P \in \mathfrak{P}} E\left(P, \operatorname{pr}^{P} \phi, s\right)
$$

where $\operatorname{pr}^{P}$ denotes the orthogonal projection onto $V^{P}$, and

$$
E^{\mathrm{cst}}(\phi, s)(g)=\left(E^{P}(\phi, s)\left(g_{P}^{-1} g\right)\right)_{P \in \mathfrak{P}} .
$$

Here, the constant term of $E^{P}(\phi, s)$ is defined by

$$
E^{P}(\phi, s)(g):=\operatorname{vol}\left(\Gamma_{N} \backslash N\right)^{-1} \int_{\Gamma_{N} \backslash N} \operatorname{pr}^{P} E(\phi, s)(n g) d n
$$

for $N=N_{P}$. Then we have

$$
E^{\mathrm{cst}}(\phi, s)=\phi_{s}+(C(s) \phi)_{1-s}
$$

where $C(s)$ is the scattering operator acting on $\mathrm{H} \otimes V^{\mathrm{cst}}$.

Now let us describe the spectral decomposition of $L^{2}(\Gamma \backslash G, \chi)$,

$$
L^{2}(\Gamma \backslash G, \chi)=L^{2}(\Gamma \backslash G, \chi)_{\text {cus }} \oplus L^{2}(\Gamma \backslash G, \chi)_{\text {res }} \oplus L^{2}(\Gamma \backslash G, \chi)_{\mathrm{ct}} .
$$

Here $L^{2}(\Gamma \backslash G, \chi)_{\text {cus }}$ is the space of the cusp forms in $L^{2}(\Gamma \backslash G, \chi)$, and decomposes into a Hilbert direct sum of closed irreducible $G$-invariant subspaces with finite multiplicities. The residual part $L^{2}(\Gamma \backslash G, \chi)_{\text {res }}$ is the direct sum of the constants and of finitely many copies of the complementary series representation of $G$ such that some Eisenstein series has a pole at $s \in\left(\frac{1}{2}, 1\right]$. These two spaces constitute the discrete part $L^{2}(\Gamma \backslash G, \chi)_{\text {dis. }}$. The continuous part $L^{2}(\Gamma \backslash G, \chi)_{\text {ct }}$ is isometric to

$$
\left\{\Phi \in L^{2}\left(\frac{1}{2}+i \mathbb{R}, \frac{d \tau}{4 \pi}\right) \hat{\otimes} \mathbf{H} \otimes V^{\mathrm{cst}} \mid \Phi\left(\frac{1}{2}-i \tau\right)=C\left(\frac{1}{2}+i \tau\right) \Phi\left(\frac{1}{2}+i \tau\right)\right\}
$$

by

$$
\mathcal{I}^{\mathrm{ct}} \Phi=\frac{1}{4 \pi} \int_{-\infty}^{\infty} E\left(\Phi, \frac{1}{2}+i \tau\right) d \tau
$$

where $E\left(\Phi, \frac{1}{2}+i \tau\right)$ is defined as in (4.2) with $\phi=\Phi$ and $s=\frac{1}{2}+i \tau$. For $f \in L^{1}(G)$, we define a representation on $L^{2}(\Gamma \backslash G, \chi)$ by

$$
\pi(f):=\int_{G} f(g) \pi(g) d g
$$

where $\pi$ is the right translation action given by $(\pi(g) \phi)(x)=\phi(x g)$ for $\phi \in L^{2}(\Gamma \backslash G, \chi)$. We put $\pi^{\text {dis }}(f)=\operatorname{pr}^{\text {dis }} \circ \pi(f), \pi^{\text {ct }}(f)=\operatorname{pr}^{\text {ct }} \circ \pi(f)$ where pr ${ }^{\text {dis }}$, pr $^{\text {ct }}$ denote the orthogonal projections onto $L^{2}(\Gamma \backslash G, \chi)_{\text {dis }}, L^{2}(\Gamma \backslash G, \chi)_{\text {ct }}$, respectively. In particular, $\pi^{\text {ct }}(f)$ intertwines with $\pi_{\frac{1}{2}+i \tau}^{\text {cst }}(f):=$ $\pi_{\frac{1}{2}+i \tau}(f) \otimes \operatorname{Id}_{V^{\text {cst }}}$ by $\mathcal{I}^{\text {ct }}$ given in $(4.3)$. 


\section{P. Loya, S. Moroianu And J. PARK}

Since $\widetilde{D}_{r}$ is a left invariant differential operator, there is a function $\hat{f}_{t, r} \in C^{\infty}(G, M(2, \mathbb{C}))$ such that

$$
\hat{f}_{t, r}\left(x^{-1} y\right)=\left(\widetilde{D}_{r} e^{-t \widetilde{D}_{r}^{2}}\right)(x, y) \quad \text { for } x, y \in G .
$$

By the heat kernel estimates in [Don79], which also holds for the generalized Laplacian $\widetilde{D}_{r}^{2}$ with the form in (2.3), we have

$$
\left\|d_{t}^{i} d_{x}^{j} d_{y}^{k} \hat{f}_{t, r}\left(x^{-1} y\right)\right\| \leqslant C t^{-\frac{5}{2}-i-j-k} \exp \left(-\frac{d_{G}^{2}(x, y)}{4 t}\right)
$$

where $C$ is a positive constant and $d_{G}$ is the metric over $G$. (Note that we apply the method in [Don79] to a certain co-compact discrete subgroup $\Gamma^{\prime}$ in $G$ to obtain the above estimate.) Put $f_{t, r}:=\operatorname{tr}\left(\hat{f}_{t, r}\right)$ where 'tr' denotes the local trace over $M(2, \mathbb{C})$. Then the estimate (4.4) implies that $f_{t, r}$ lies in the Harish-Chandra $L^{1}$-Schwartz space $\mathcal{C}^{1}(G)\left(\subset L^{1}(G)\right)$ defined by

$$
\mathcal{C}^{1}(G)=\left\{f \in C^{\infty}(G)|| f\left(D_{1} k_{\theta_{1}} a_{u} k_{\theta_{2}} D_{2}\right) \mid \leqslant C e^{-|u|}\left(1+|u|+\left|\theta_{1}+\theta_{2}\right|\right)^{-n}, \quad \forall n \in \mathbb{N}, D_{1}, D_{2} \in \mathfrak{g}\right\},
$$

where $f\left(D_{1} k_{\theta_{1}} a_{u} k_{\theta_{2}} D_{2}\right)$ denotes the convolution $D_{1} * \delta_{k_{\theta_{1}}} * \delta_{a_{u}} * \delta_{k_{\theta_{2}}} * D_{2}$ evaluated on $f$. Let us put

$$
K(t, x, y):=\sum_{\gamma \in Z \backslash \Gamma} \hat{f}_{t, r}\left(x^{-1} \gamma y\right) \chi(\gamma)=\sum_{\gamma \in Z \backslash \Gamma} \widetilde{D}_{r} e^{-t \widetilde{D}_{r}^{2}}(x, \gamma y) \chi(\gamma) \quad \text { for } x, y \in G,
$$

which is absolutely uniformly convergent on compact sets in $G$. For a $\Gamma$-cuspidal parabolic subgroup $P_{j}=P=N A Z$, we define the constant term of $K(t, x, y)$ along $P$ as follows,

$$
K_{P}(t, x, y)=\operatorname{vol}\left(\Gamma_{N} \backslash N\right)^{-1} \int_{\Gamma_{N} \backslash N} \sum_{\gamma \in Z \backslash \Gamma_{P}} \hat{f}_{t, r}\left(x^{-1} \gamma n y\right) \chi(\gamma) \operatorname{pr}^{P} d n .
$$

For $u \in \mathbb{R}$, let $\alpha_{P}(u)$ be the characteristic function of $\left\{x \in G \mid H_{P}(x)+u_{P}>u\right\}$, which projects on certain region $\mathcal{C}_{P, u} \subset \Gamma \backslash G$ for a large $u$. Then the truncation of $K(x, x)$ is defined by

$$
\Lambda_{u} K(t, x, x):=K(t, x, x)-\sum_{P \in \mathfrak{P}} \alpha_{P}(u) K_{P}(t, x, x),
$$

which is an automorphic form over $\Gamma \backslash G$.

Proposition 4.1 (Maass-Selberg relation). For $u \gg 0$, we have

$$
\begin{aligned}
\int_{\Gamma \backslash G} \operatorname{tr}\left(\Lambda_{u} K(t, x, x)\right) d x= & u \frac{1}{2 \pi} \int_{-\infty}^{\infty} \operatorname{Tr}\left(\pi_{\frac{1}{2}+i \tau}^{\mathrm{cst}}\left(f_{t, r}\right)\right) d \tau+\operatorname{Tr}\left(\pi^{\mathrm{dis}}\left(f_{t, r}\right)\right)+\frac{1}{4} \operatorname{Tr}\left(C\left(\frac{1}{2}\right) \pi_{\frac{1}{2}}^{\mathrm{cst}}\left(f_{t, r}\right)\right) \\
& -\frac{1}{4 \pi} \int_{-\infty}^{\infty} \operatorname{Tr}\left(C^{\prime}\left(\frac{1}{2}-i \tau\right) C\left(\frac{1}{2}+i \tau\right) \pi_{\frac{1}{2}+i \tau}^{\mathrm{cst}}\left(f_{t, r}\right)\right) d \tau .
\end{aligned}
$$

Proof. For a test function with compact support and $K$-finite condition, we can prove this proposition by simply following the argument in [Hof94, pp. 58-60]. Then this can be generalized easily to our test function $f_{t, r}$ as in [Hof94, Proof of the Theorem 25]. The finiteness of the integrand of the integrals on the right-hand side follows from Theorem 1.1 and Lemma 5.2 since $\operatorname{Tr}\left(\pi_{\frac{1}{2}+i \tau}^{\text {cst }}\left(f_{t, r}\right)\right)$ is given by $\kappa^{\mathrm{t}}$-copies of $\Theta_{\frac{1}{2}+i \tau}\left(f_{t, r}\right):=\operatorname{Tr}\left(\pi_{\frac{1}{2}+i \tau}\left(f_{t, r}\right)\right)$ recalling $\pi_{\frac{1}{2}+i \tau}^{\text {cst }}\left(f_{t, r}\right):=\pi_{\frac{1}{2}+i \tau}\left(f_{t, r}\right) \otimes \operatorname{Id}_{V^{\text {cst }}}$.

From Proposition 4.1, one can see that the first term on the right-hand side of (4.5) is blowing up as $u \rightarrow \infty$. Hence, it is natural to remove this term in the following definition,

$$
\begin{aligned}
\operatorname{Tr}\left(D_{r} e^{-t D_{r}^{2}}\right):=\operatorname{Tr}\left(\pi^{\mathrm{dis}}\left(f_{t, r}\right)\right)+\frac{1}{4} \operatorname{Tr}\left(C\left(\frac{1}{2}\right) \pi_{\frac{1}{2}}^{\mathrm{cst}}\left(f_{t, r}\right)\right) \\
-\frac{1}{4 \pi} \int_{-\infty}^{\infty} \operatorname{Tr}\left(C^{\prime}\left(\frac{1}{2}-i \tau\right) C\left(\frac{1}{2}+i \tau\right) \pi_{\frac{1}{2}+i \tau}^{\mathrm{cst}}\left(f_{t, r}\right)\right) d \tau .
\end{aligned}
$$




\section{AdiABATIC LIMIT OF THE ETA INVARIANT}

This regularized trace is essentially the same as the $b$-trace of Melrose [Mel93], and is related with the geometric side of the Selberg trace formula as we will see in Proposition 4.2. Denote

$$
h(\tau)=\Theta_{\frac{1}{2}+i \tau}(f), \quad h(n)=\Theta_{n}(f)
$$

where $\Theta_{\frac{1}{2}+i \tau}(f):=\operatorname{Tr}\left(\pi_{\frac{1}{2}+i \tau}(f)\right)$ for a principal series representation $\pi_{\frac{1}{2}+i \tau}$, and $\Theta_{n}(f):=\operatorname{Tr}\left(\pi_{n}(f)\right)$ for a discrete series representation $\pi_{n}$. An operator $J(s)$ over $\mathrm{H}=L^{2}(Z \backslash K)$ is defined by

$$
J(s) \phi_{m}=\frac{1}{\sqrt{\pi}} \cdot \frac{\Gamma(s) \Gamma\left(s-\frac{1}{2}\right)}{\Gamma(s+m / 2) \Gamma(s-m / 2)} \phi_{m}
$$

for the basis $\phi_{m}\left(k_{\theta}\right)=e^{i m \theta} \in \mathrm{H}$ where $k_{\theta}=\left(\begin{array}{c}\cos \theta \sin \theta \\ -\sin \theta \cos \theta\end{array}\right)$. The following proposition follows from [Hof94, Theorem 13 and Lemma 24].

Proposition 4.2 (Selberg trace formula). We have

$$
\begin{aligned}
\operatorname{Tr}\left(D_{r} e^{-t D_{r}^{2}}\right)= & \frac{\operatorname{vol}(\Gamma \backslash G)}{2 \pi}\left(\int_{-\infty}^{\infty} \tau \tanh (\pi \tau) h_{t, r}(\tau) d \tau+\sum_{n \equiv 0(\bmod 2)}(|n|-1) h_{t, r}(n)\right) \\
& +\sum_{[\gamma] \in Z \backslash \Gamma_{\text {hyp }}} \frac{\operatorname{tr}(\chi(\gamma)) u_{\gamma}}{4 \pi\left[\Gamma_{\gamma}: Z\right] \sinh \left(u_{\gamma} / 2\right)} \int_{-\infty}^{\infty} \cos \left(u_{\gamma} \tau\right) h_{t, r}(\tau) d \tau \\
& -2 \kappa^{\mathrm{t}}\left(\frac{1}{2 \pi} \int_{-\infty}^{\infty} \psi(1+2 i \tau) h_{t, r}(\tau) d \tau+\frac{1}{2} \sum_{n \equiv 0(\bmod 2)} h_{t, r}(n)\right) \\
& +2\left(\kappa-\kappa^{\mathrm{t}}\right) \frac{\log 2}{2 \pi} \int_{-\infty}^{\infty} h_{t, r}(\tau) d \tau \\
& +\frac{\kappa^{\mathrm{t}}}{2} h_{t, r}(0)-\frac{1}{4 \pi} \mathrm{p} \cdot \mathrm{v} \cdot \int_{-\infty}^{\infty} \operatorname{Tr}\left(J\left(\frac{1}{2}+i \tau\right)^{-1} J^{\prime}\left(\frac{1}{2}+i \tau\right) \pi_{\frac{1}{2}+i \tau}\left(f_{t, r}\right)\right) d \tau
\end{aligned}
$$

where $h_{t, r}(\tau), h_{t, r}(n)$ are defined for $f_{t, r}$, the sum $\sum_{[\gamma] \in \Gamma_{\text {hyp }}}$ is given over the $\Gamma$-conjugacy class of hyperbolic elements $\gamma$ conjugate to $a_{u_{\gamma}}$, and $\psi(z)=\Gamma^{\prime}(z) \Gamma(z)^{-1}$.

\section{Fourier transforms $h_{t, r}(\tau), h_{t, r}(n)$}

In this section, we compute $h_{t, r}(\tau), h_{t, r}(n)$ which are needed to analyze the right-hand side of the Selberg trace formula.

First, let us consider $h_{t, r}(\tau)$. For this, recall

$$
h_{t, r}(\tau)=\operatorname{Tr}\left(\pi_{\frac{1}{2}+i \tau}\left(f_{t, r}\right)\right)=\sum_{n=1}^{\infty} \int_{G} f_{t, r}(g)\left(\pi_{\frac{1}{2}+i \tau}(g) \xi_{n}, \xi_{n}\right) d g,
$$

where $\left\{\xi_{n}\right\}_{n=1}^{\infty}$ is the orthonormal basis of the representation space of $\pi_{\frac{1}{2}+i \tau}$, which is given by the union of the following spaces indexed by $m \in \mathbb{Z}$ for $s=\frac{1}{2}+i \tau$,

$$
\mathrm{H}(s, m):=\left\{\phi_{s} \in \mathrm{H}_{s} \mid \phi_{s}\left(n a_{u} k_{\theta}\right)=e^{s u} e^{i m \theta} \quad \text { for } n a_{u} k_{\theta} \in N_{0} A_{0} K\right\} .
$$

Since $\widetilde{D}_{r}$ is $Z$-invariant, the Fourier transform $h_{t, r}(\tau)$ is non-trivial only if $m$ is an even number. Recalling

$$
\widetilde{D}_{r}=\left(\frac{2-r^{2}}{2 r}\right)+\left(\begin{array}{cc}
r^{-1} Z & 2 X_{-} \\
-2 X_{+} & -r^{-1} Z
\end{array}\right)
$$

the problem is again reduced to the following lemma, which can be obtained applying the equalities in $(3.3)$. 
LEMma 5.1. We have

$$
Z f=m f, \quad X_{ \pm} f=-\frac{i}{2}(m \pm 2 s) e^{ \pm 2 i \theta} f \quad \text { for } f \in \mathrm{H}(s, m) .
$$

The second equality in Lemma 5.1 implies that $X_{ \pm}$maps $\mathrm{H}(s, m)$ to $\mathrm{H}(s, m \pm 2)$. From these facts,

$$
\widetilde{D}_{r}\left(\begin{array}{c}
\phi_{\tau, m-2} \\
\phi_{\tau, m}
\end{array}\right)=\left(\begin{array}{cc}
r^{-1}(m-2)+2^{-1} \ell & -i(m-1-2 i \tau) \\
i(m-1+2 i \tau) & -r^{-1} m+2^{-1} \ell
\end{array}\right)\left(\begin{array}{c}
\phi_{\tau, m-2} \\
\phi_{\tau, m}
\end{array}\right),
$$

where $\ell=\left(2-r^{2}\right) / r$ and $\phi_{\tau, m-2} \in \mathrm{H}\left(\frac{1}{2}+i \tau, m-2\right), \phi_{\tau, m} \in \mathrm{H}\left(\frac{1}{2}+i \tau, m\right)$. Hence, the action of $\widetilde{D}_{r}$ on $\mathrm{H}\left(\frac{1}{2}+i \tau, m-2\right) \oplus \mathbf{H}\left(\frac{1}{2}+i \tau, m\right)$ is given by the roots of

$$
\lambda^{2}+r \lambda+\frac{r^{2}}{4}-\frac{(m-1)^{2}}{r^{2}}=(m-1)^{2}+4 \tau^{2},
$$

that is,

$$
\lambda_{ \pm}(\tau, m)=-\frac{r}{2} \pm\left((m-1)^{2}\left(1+r^{-2}\right)+4 \tau^{2}\right)^{1 / 2} \quad \text { for } m \in 2 \mathbb{Z}, \tau \in \mathbb{R}^{+} .
$$

Therefore, we have the following result.

LEMma 5.2. We have

$$
h_{t, r}(\tau)=\Theta_{\frac{1}{2}+i \tau}\left(f_{t, r}\right)=\sum_{m \in 2 \mathbb{Z}}\left(\lambda_{+}(\tau, m) e^{-t \lambda_{+}(\tau, m)^{2}}+\lambda_{-}(\tau, m) e^{-t \lambda_{-}(\tau, m)^{2}}\right) .
$$

Just repeating the above computation applied to the Eisenstein series $E\left(P, \phi_{m}, s\right)$, we can also prove Theorem 1.1.

Next we compute $h_{t, r}(n)$ for the discrete series representation $\pi_{n}$. We review the discrete series representations of $G=\operatorname{SL}(2, \mathbb{R})$. For this it is more convenient to use the Lie group $\mathrm{SU}(1,1)$ which is conjugate to $\mathrm{SL}(2, \mathbb{R})$ within $\mathrm{SL}(2, \mathbb{C})$ :

$$
\left(\begin{array}{ll}
1 & i \\
i & 1
\end{array}\right) \operatorname{SU}(1,1)\left(\begin{array}{ll}
1 & i \\
i & 1
\end{array}\right)^{-1}=\operatorname{SL}(2, \mathbb{R})
$$

Here

$$
\mathrm{SU}(1,1)=\left\{\left.\left(\begin{array}{cc}
\alpha & \beta \\
\bar{\beta} & \bar{\alpha}
\end{array}\right)|| \alpha\right|^{2}-|\beta|^{2}=1\right\} .
$$

Then the holomorphic discrete series $\pi_{n}(n \in \mathbb{N})$ as a representation of $\mathrm{SU}(1,1)$ acts on analytic functions on the disc by

$$
\pi_{n}\left(\begin{array}{ll}
\frac{\alpha}{\beta} & \bar{\alpha}
\end{array}\right) f(z)=(-\beta z+\bar{\alpha})^{-n} f\left(\frac{\alpha z-\bar{\beta}}{-\beta z+\bar{\alpha}}\right),
$$

and the norm, except for a constant factor, is given by

$$
\|f\|= \begin{cases}\int_{|z|<1}|f(z)|^{2}\left(1-|z|^{2}\right)^{n-2} d z d \bar{z} & \text { for } n \geqslant 2, \\ \sup _{0 \leqslant r<1} \int_{0}^{2 \pi}\left|f\left(r e^{i \theta}\right)\right|^{2} d \theta & \text { for } n=1 .\end{cases}
$$

The anti-holomorphic discrete series $\pi_{n}(n \in-\mathbb{N})$ as a representation of $\mathrm{SU}(1,1)$ acts on analytic functions on the disc by

$$
\pi_{n}\left(\begin{array}{ll}
\frac{\alpha}{\beta} & \bar{\alpha}
\end{array}\right) f(z)=(-\bar{\beta} z+\alpha)^{-n} f\left(\frac{\bar{\alpha} z-\beta}{-\bar{\beta} z+\alpha}\right)
$$

with the same norm. We refer to [Kna01, ch. II, $\S \S 5$ and 6] for a nice introduction on the discrete series $\pi_{n}$ of $\mathrm{SL}(2, \mathbb{R})$. 


\section{AdiABATIC LIMIT OF THE ETA INVARIANT}

Lemma 5.3. For the basis $\left\{z^{N}\right\}_{N \in\{0\} \cup \mathbb{N}}$ of the space of analytic functions on the disc, we have

$$
X_{+} z^{N}=(N+n) z^{N+1}, \quad X_{-} z^{N}=-N z^{N-1}, \quad Z z^{N}=(2 N+n) z^{N}
$$

by the action of $\pi_{n}, n \in \mathbb{N}$, and

$$
X_{+} z^{N}=-N z^{N+1}, \quad X_{-} z^{N}=(N+n) z^{N-1}, \quad Z z^{N}=-(2 N+n) z^{N}
$$

by the action of $\pi_{n}, n \in-\mathbb{N}$.

Proof. By elementary computations, we can see that the subgroups generating $K, H, A$ are transformed as follows:

$$
\begin{aligned}
\left(\begin{array}{cc}
\cos \theta & \sin \theta \\
-\sin \theta & \cos \theta
\end{array}\right) & \longrightarrow\left(\begin{array}{cc}
e^{i \theta} & 0 \\
0 & e^{-i \theta}
\end{array}\right) \\
\left(\begin{array}{cc}
e^{t} & 0 \\
0 & e^{-t}
\end{array}\right) & \longrightarrow\left(\begin{array}{cc}
\cosh t & i \sinh t \\
-i \sinh t & \cosh t
\end{array}\right), \\
\left(\begin{array}{cc}
\cosh t & \sinh t \\
\sinh t & \cosh t
\end{array}\right) & \longrightarrow\left(\begin{array}{cc}
\cosh t & \sinh t \\
\sinh t & \cosh t
\end{array}\right),
\end{aligned}
$$

where the matrices on the right-hand side denote elements in $\mathrm{SU}(1,1)$. To see the action of $K$ under $\pi_{n}$ for $n \in \mathbb{N}$, let us consider

$$
\pi_{n}\left(\begin{array}{cc}
e^{i \theta} & 0 \\
0 & e^{-i \theta}
\end{array}\right) z^{N}=e^{(2 N+n) i \theta} z^{N}
$$

which implies

$$
Z z^{N}=-\left.i \frac{d}{d \theta}\right|_{\theta=0} \pi_{n}\left(\begin{array}{cc}
e^{i \theta} & 0 \\
0 & e^{-i \theta}
\end{array}\right) z^{N}=(2 N+n) z^{N}
$$

In a similar way, we can show that the action $\pi_{n}$ for $n \in \mathbb{N}$ by $H, A$ are given by

$$
H z^{N}=i(N+n) z^{N+1}+i N z^{N-1}, \quad A z^{N}=(N+n) z^{N+1}-N z^{N-1} .
$$

These imply the equalities for $\pi_{n}$ for $n \in \mathbb{N}$. The case for $\pi_{n}$ for $n \in-\mathbb{N}$ can be obtained by taking the complex conjugates of equalities for the action of $\pi_{n}$ for $n \in \mathbb{N}$.

Now we consider the action of $\widetilde{D}_{r}$ under $\pi_{n}$ for $n \in \mathbb{N}$. From (5.3), we can see that $z^{N}$ are the $K$-type vectors of weight $m$ if $m=2 N+n$. By Lemma 5.3, over $(\alpha, \beta)$ for $K$-type $(m-2)$, $m$ vectors $\alpha, \beta$ in the representation space of $\pi_{n}$, the Dirac operator $\widetilde{D}_{r}$ acts by

$$
\left(\frac{2-r^{2}}{2 r}\right) \operatorname{Id}+\left(\begin{array}{cc}
r^{-1}(m-2) & n-m \\
-(n+m-2) & -r^{-1} m
\end{array}\right)
$$

noting $N=(m-n) / 2$. We have two cases. First, if $\beta$ is not the minimal $K$-type for $\pi_{n}$, that is, $m \gtrless n$, as in the derivation of (5.2), we can obtain the corresponding eigenvalue equation

$$
\lambda^{2}+r \lambda+\frac{r^{2}}{4}-\frac{(m-1)^{2}}{r^{2}}=(m-1)^{2}-(n-1)^{2} .
$$

Hence,

$$
\lambda_{ \pm}(n, m)=-\frac{r}{2} \pm\left((m-1)^{2}\left(1+r^{-2}\right)-(n-1)^{2}\right)^{1 / 2} \quad \text { for } m=n+2, n+4, \ldots
$$

Second, if $\beta$ is the minimal $K$-type for $\pi_{n}$, that is, $K$-type $m=n$ vector, then $\alpha$ is just trivial. Hence, the eigenvalue is given by

$$
\lambda(n)=-\frac{r}{2}+\frac{1-n}{r} .
$$




\section{P. Loya, S. Moroianu And J. PARK}

Repeating the same procedure as in the case of $\pi_{n}$ for $n \in \mathbb{N}$, we can obtain the same eigenvalues $\lambda_{ \pm}(n, m), \lambda(n)$ for $\pi_{n}$ for $n \in-\mathbb{N}$. In our case, $n$ should be an even number since $\Gamma$ contains $-\mathrm{Id}$. Combining all of the facts derived in the above, we have the following result.

Lemma 5.4. For $n \in 2 \mathbb{N}, h(n)=\Theta_{n}(f)$, we have

$$
h_{t, r}(n)=h_{t, r}(-n)=\left(\lambda(n) e^{-t \lambda(n)^{2}}+\sum_{m \in n+2 \mathbb{N}}\left(\lambda_{+}(n, m) e^{-t \lambda_{+}(n, m)^{2}}+\lambda_{-}(n, m) e^{-t \lambda_{-}(n, m)^{2}}\right)\right)
$$

where

$$
\lambda(n)=-\frac{r}{2}+\frac{1-n}{r}, \quad \lambda_{ \pm}(n, m)=-\frac{r}{2} \pm\left((m-1)^{2}\left(1+r^{-2}\right)-(n-1)^{2}\right)^{1 / 2} .
$$

\section{Eta function of $D_{r}$ : principal series part}

Now we study the eta function defined by

$$
\eta\left(D_{r}, s\right):=\frac{1}{\Gamma((s+1) / 2)} \int_{0}^{\infty} t^{(s-1) / 2} \operatorname{Tr}\left(D_{r} e^{-t D_{r}^{2}}\right) d t
$$

for $\Re(s) \gg 0$ and $r$ near 0 . First let us recall that the bottom of each branch of continuous spectrum of $D_{r}$ goes to $\infty$ as $r \rightarrow 0$ by Theorem 1.1. Hence, for a small $r>0, \operatorname{Tr}\left(D_{r} e^{-t D_{r}^{2}}\right)$ decays exponentially as $t \rightarrow \infty$. To analyze $\operatorname{Tr}\left(D_{r} e^{-t D_{r}^{2}}\right)$ in more detail, we apply Proposition 4.2 which relates $\operatorname{Tr}\left(D_{r} e^{-t D_{r}^{2}}\right)$ with the geometric side. Note that this geometric side can be decomposed into two parts:

$$
\begin{aligned}
\operatorname{Tr}_{\mathrm{p}}\left(D_{r} e^{-t D_{r}^{2}}\right) & =\operatorname{Tr}\left(D_{r} e^{-t D_{r}^{2}}\right)-\operatorname{Tr}_{\mathrm{d}}\left(D_{r} e^{-t D_{r}^{2}}\right), \\
\operatorname{Tr}_{\mathrm{d}}\left(D_{r} e^{-t D_{r}^{2}}\right) & =\frac{\operatorname{vol}(\Gamma \backslash G)}{2 \pi} \sum_{n \equiv 0(\bmod 2)}(|n|-1) h_{t, r}(n)-\kappa^{\mathrm{t}} \sum_{n \equiv 0(\bmod 2)} h_{t, r}(n)
\end{aligned}
$$

and accordingly we also decompose the eta function $\eta\left(D_{r}, s\right)$ into

$$
\eta\left(D_{r}, s\right)=\eta_{\mathrm{p}}\left(D_{r}, s\right)+\eta_{\mathrm{d}}\left(D_{r}, s\right) .
$$

The principal part of the eta function $\eta_{\mathrm{p}}\left(D_{r}, s\right)$ is studied in this section and the other part $\eta_{\mathrm{d}}\left(D_{r}, s\right)$ will be considered in the next section.

We start with the following lemma.

Lemma 6.1. Putting $I(m, r, \tau)=\left((m-1)^{2}\left(1+r^{-2}\right)+4 \tau^{2}\right)^{\frac{1}{2}}$,

$$
h_{t, r}(\tau)=\exp \left(-\frac{r^{2}}{4} t-4 \tau^{2} t\right) \cdot \sum_{m \in 2 \mathbb{Z}} e^{-(m-1)^{2}\left(1+r^{-2}\right) t}\left(\sum_{k=0}^{\infty}\left(-r \frac{(r t)^{2 k}}{(2 k) !}+2 \frac{(r t)^{2 k-1}}{(2 k-1) !}\right) I(m, r, \tau)^{2 k}\right)
$$

where the term $(r t)^{2 k-1} /(2 k-1)$ ! for $k=0$ vanishes and for $t \in[0,1]$ and $r \in(0,1]$ the following estimate holds,

$$
\left|h_{t, r}(\tau)\right| \leqslant 2 r \exp \left(-\frac{r^{2}}{4} t-4 \tau^{2} t\right) \cdot \sum_{m \in 2 \mathbb{Z}} e^{-(m-1)^{2}\left(1+r^{-2}\right) t}\left(1+I(m, r, \tau)^{2}+e^{I(m, r, \tau)^{2} r t}\right) .
$$

Proof. We can rewrite $h_{t, r}(\tau)$ as follows,

$$
\begin{aligned}
h_{t, r}(\tau)= & \exp \left(-\frac{r^{2}}{4} t-4 \tau^{2} t\right) \sum_{m \in 2 \mathbb{Z}} e^{-(m-1)^{2}\left(1+r^{-2}\right) t} \\
& \cdot\left(-\frac{r}{2}\left(e^{I(m, r, \tau) r t}+e^{-I(m, r, \tau) r t}\right)+I(m, r, \tau)\left(e^{I(m, r, \tau) r t}-e^{-I(m, r, \tau) r t}\right)\right) .
\end{aligned}
$$




\section{Adiabatic Limit of THE ETA INVARIANT}

Now the Taylor expansion of $e^{I(m, r, \tau) r t} \pm e^{-I(m, r, \tau) r t}$ gives us the claimed form of the first equality. To prove the second estimate, we note that

$$
\begin{aligned}
& \sum_{k=0}^{\infty}\left(-r \frac{(r t)^{2 k}}{(2 k) !}+2 \frac{(r t)^{2 k-1}}{(2 k-1) !}\right) I(m, r, \tau)^{2 k} \\
& \quad=-r+r t\left(2-\frac{r^{2} t}{2}\right) I(m, r, \tau)^{2}+\sum_{k=2}^{\infty} \frac{(r t)^{2 k-1}}{(2 k-1) !}\left(2-\frac{r^{2} t}{2 k}\right) I(m, r, \tau)^{2 k} .
\end{aligned}
$$

For $t \in[0,1]$ and $r \in(0,1]$, observe that

$$
\sum_{k=2}^{\infty} \frac{(r t)^{2 k-1}}{(2 k-1) !}\left(2-\frac{r^{2} t}{2 k}\right) I(m, r, \tau)^{2 k} \leqslant 2 r \sum_{k=0}^{\infty} \frac{(r t)^{k}}{k !} I(m, r, \tau)^{2 k},
$$

from which it is easy to derive the estimate.

Our first task in this section is to obtain the asymptotic expansion of $\operatorname{Tr}_{\mathrm{p}}\left(D_{r} e^{-t D_{r}^{2}}\right)$ as $t \rightarrow 0$. By Lemma 6.1, we can rewrite the first part of $\operatorname{Tr}_{\mathrm{p}}\left(D_{r} e^{-t D_{r}^{2}}\right)$ from (4.8) as follows,

$$
\begin{aligned}
\int_{-\infty}^{\infty} \tau \tanh (\pi \tau) h_{t, r}(\tau) d \tau= & \sum_{m \in 2 \mathbb{Z}} \exp \left(-\frac{r^{2}}{4} t-(m-1)^{2}\left(1+r^{-2}\right) t\right) \\
& \cdot \sum_{k=0}^{\infty} \sum_{k=p+q}\left(a_{k, p, q}(r) t^{2 k}+b_{k, p, q}(r) t^{2 k-1}\right) \\
& \cdot(m-1)^{2 p}\left(1+r^{-2}\right)^{p} \int_{-\infty}^{\infty} \tau \tanh (\pi \tau)(2 \tau)^{2 q} e^{-4 \tau^{2} t} d \tau
\end{aligned}
$$

Here the coefficients $a_{k, p, q}(r), b_{k, p, q}(r)$ are given by

$$
a_{k, p, q}(r)=-\left(\begin{array}{l}
k \\
p
\end{array}\right) \frac{r^{2 k+1}}{(2 k) !}, \quad b_{k, p, q}(r)=2\left(\begin{array}{l}
k \\
p
\end{array}\right) \frac{r^{2 k-1}}{(2 k-1) !} \quad\left(\text { with } b_{0, p, q}(r)=0\right),
$$

which are of order $\mathrm{O}(r)$ uniformly for small $r>0$. The integral in the last line can be handled as follows,

$$
\begin{aligned}
\int_{-\infty}^{\infty} \tau \tanh (\pi \tau)(2 \tau)^{2 q} e^{-4 \tau^{2} t} d \tau & =(-1)^{q} \partial_{t}^{q} \int_{-\infty}^{\infty} \tau \tanh (\pi \tau) e^{-4 \tau^{2} t} d \tau \\
& =(-1)^{q} \partial_{t}^{q} \int_{0}^{\infty} \tanh (\pi \sqrt{x}) e^{-4 t x} d x \\
& =(-1)^{q} \partial_{t}^{q} \frac{\pi}{8 t} \int_{0}^{\infty}\left(\sum_{k=0}^{\infty} \frac{(-4 t x)^{k}}{k !}\right) \frac{\cosh ^{-2}(\pi \sqrt{x})}{\sqrt{x}} d x
\end{aligned}
$$

Hence, we have

$$
\int_{-\infty}^{\infty} \tau \tanh (\pi \tau)(2 \tau)^{2 q} e^{-4 \tau^{2} t} d \tau \sim \sum_{k=0}^{\infty} a_{k} t^{-q-1+k} \quad \text { as } t \rightarrow 0
$$

where $a_{k}$ are independent of $r$. Now we note the following equalities for the first and the third factors on the right-hand side of (6.3),

$$
\begin{gathered}
\sum_{m \in 2 \mathbb{Z}}(m-1)^{2 p}\left(1+r^{-2}\right)^{p} \exp \left(-(m-1)^{2}\left(1+r^{-2}\right) t\right) \\
=(-1)^{p} \partial_{t}^{p} \sum_{m \in \mathbb{Z}} \exp \left(-4\left(m-\frac{1}{2}\right)^{2}\left(1+r^{-2}\right) t\right)
\end{gathered}
$$




$$
\begin{aligned}
& =(-1)^{p} \partial_{t}^{p} \sum_{m \in \mathbb{Z}}(-1)^{m} \frac{\sqrt{\pi}}{2 \sqrt{\left(1+r^{-2}\right) t}} \exp \left(-\frac{\pi^{2} m^{2}}{4\left(1+r^{-2}\right) t}\right) \\
& =(-1)^{p} \partial_{t}^{p}\left(\frac{\sqrt{\pi} r}{2 \sqrt{\left(1+r^{2}\right) t}}+\sum_{m \in \mathbb{Z}-\{0\}}(-1)^{m} \frac{\sqrt{\pi}}{2 \sqrt{\left(1+r^{-2}\right) t}} \exp \left(-\frac{\pi^{2} m^{2}}{4\left(1+r^{-2}\right) t}\right)\right),
\end{aligned}
$$

where the second equality is the Poisson summation formula. Note that the terms for non-zero $m$ in the last line of (6.5) decays exponentially as $t \rightarrow 0$, so that small time asymptotics is given by the first term in the last line of (6.5). Therefore, we have

$$
\sum_{m \in 2 \mathbb{Z}}(m-1)^{2 p}\left(1+r^{-2}\right)^{p} \exp \left(-(m-1)^{2}\left(1+r^{-2}\right) t\right) \sim a(r) t^{-\frac{1}{2}-p} \quad \text { as } t \rightarrow 0
$$

where $a(r)$ is given by $r / \sqrt{1+r^{2}}$ up to a constant, hence it is of $\mathrm{O}(r)$ for small $r>0$.

By (6.3) and the asymptotic expansions in (6.4), (6.6), taking care of the $r$-dependence of their coefficients, we can conclude that

$$
\int_{-\infty}^{\infty} \tau \tanh (\pi \tau) h_{t, r}(\tau) d \tau \sim \sum_{k=0}^{\infty} a_{k}(r) t^{-\frac{3}{2}+k} \quad \text { as } t \rightarrow 0
$$

where $a_{k}(r)$ depends only on $r$ and is of $\mathrm{O}\left(r^{2}\right)$ for small $r>0$ uniformly with respect to the index $k$.

Now for the second part of $\operatorname{Tr}_{\mathrm{p}}\left(D_{r} e^{-t D_{r}^{2}}\right)$, we repeat the above process and noting

$$
\int_{-\infty}^{\infty} \cos \left(u_{\gamma} \tau\right)(2 \tau)^{2 q} e^{-4 \tau^{2} t} d \tau=(-1)^{q} \partial_{t}^{q} \int_{-\infty}^{\infty} \cos \left(u_{\gamma} \tau\right) e^{-4 \tau^{2} t} d \tau=(-1)^{q} \partial_{t}^{q}\left(\frac{\sqrt{\pi}}{\sqrt{t}} \exp \left(-\frac{u_{\gamma}^{2}}{4 t}\right)\right)
$$

we can see that this term does not contribute to the asymptotics as $t \rightarrow 0$.

To deal with the third part of $\operatorname{Tr}_{\mathrm{p}}\left(D_{r} e^{-t D_{r}^{2}}\right)$, we recall

$$
\psi(1+z) \sim \log z+\frac{1}{2 z}-\sum_{k=1}^{\infty} \frac{B_{2 k}}{2 k} z^{-2 k} \quad \text { as } z \rightarrow \infty,
$$

where $B_{2 k}$ is the Bernoulli's number, which implies

$$
\int_{-\infty}^{\infty} \psi(1+2 i \tau)(2 \tau)^{2 q} e^{-4 \tau^{2} t} d \tau \sim b t^{-\frac{1}{2}-q} \log t+c+\sum_{k=0}^{\infty} a_{k} t^{-q-\frac{1}{2}+k} \quad \text { as } t \rightarrow 0
$$

where the constant $c$ vanishes unless $q=0$. Proceeding as before and using (6.9),

$$
\int_{-\infty}^{\infty} \psi(1+2 i \tau) h_{t, r}(\tau) d \tau \sim \sum_{k=0}^{\infty} a_{k}(r) t^{-1+k / 2}+b_{k}(r) t^{-1+k} \log t \quad \text { as } t \rightarrow 0,
$$

where $a_{k}(r), b_{k}(r)$ depend only on $r$ and is of $\mathrm{O}\left(r^{2}\right)$ for small $r>0$ uniformly with respect to the index $k$.

For the fourth part of $\operatorname{Tr}_{\mathrm{p}}\left(D_{r} e^{-t D_{r}^{2}}\right)$, it is also easy to obtain the following asymptotic expansion

$$
\int_{-\infty}^{\infty} h_{t, r}(\tau) d \tau \sim \sum_{k=0}^{\infty} a_{k}(r) t^{-1+k} \quad \text { as } t \rightarrow 0
$$

where $a_{k}(r)$ depends only on $r$ and is of $\mathrm{O}\left(r^{2}\right)$ for small $r>0$ uniformly with respect to the index $k$.

Now it is easy to see that the next term $\left(\kappa_{\mathrm{t}} / 2\right) h_{t, r}(0)$ contributes to the small time asymptotics by (6.7) with the first term $a_{1}(r) t^{-\frac{1}{2}}$.

By (4.7), the integrand of the last integral of the geometric side can be expressed by

$$
\psi\left(\frac{1}{2}+i \tau\right)+\psi(i \tau)-\psi\left(\frac{1+m}{2}+i \tau\right)-\psi\left(\frac{1-m}{2}+i \tau\right) .
$$




\section{AdiABATIC LIMIT OF THE ETA INVARIANT}

Using the following formulas about $\psi(z)$,

$$
\psi(z+1)=\frac{1}{z}+\psi(z), \quad \psi(z)+\psi\left(z+\frac{1}{2}\right)=2(\psi(2 z)-\log 2),
$$

the terms in (6.12) can be rewritten as

$$
2(\psi(1+i \tau)-\psi(1+2 i \tau))-\frac{1}{i \tau}+2 \log 2-4\left(\frac{1}{1+4 \tau^{2}}+\frac{3}{3^{2}+4 \tau^{2}}+\cdots+\frac{m-1}{(m-1)^{2}+4 \tau^{2}}\right) .
$$

The terms in the first line give us the asymptotics as (6.10). The terms in the second line also can be handled as in a similar way and we can show that these term gives us the asymptotics

$$
\sum_{k=0}^{\infty} a_{k}(r) t^{(-1+k) / 2} \quad \text { as } t \rightarrow 0
$$

where $a_{k}(r)$ depends only on $r$ and is of $\mathrm{O}\left(r^{2}\right)$ for small $r>0$ uniformly with respect to the index $k$. Combining (6.7), (6.10), (6.14) and facts derived in the above, we obtain the following theorem.

TheOrem 6.2. The small time asymptotics is given by

$$
\operatorname{Tr}_{\mathrm{p}}\left(D_{r} e^{-t D_{r}^{2}}\right) \sim \sum_{k=0}^{\infty} a_{k}(r) t^{(-3+k) / 2}+b_{k}(r) t^{-1+k} \log t \quad \text { as } t \rightarrow 0,
$$

where $a_{k}(r), b_{k}(r)$ depend only on $r$ and is of $\mathrm{O}\left(r^{2}\right)$ for small $r>0$ uniformly with respect to the index $k$. In particular, if $\kappa^{t}=0$, it has the following simple form,

$$
\operatorname{Tr}_{\mathrm{p}}\left(D_{r} e^{-t D_{r}^{2}}\right) \sim \sum_{k=0}^{\infty} a_{k}(r) t^{(-3+k) / 2} \quad \text { as } t \rightarrow 0 .
$$

This theorem also immediately implies the following result.

Theorem 6.3. For a sufficiently small $r>0$, the function $\eta_{\mathrm{p}}\left(D_{r}, s\right)$ defined for $\Re(s)>2$ extends meromorphically to $\mathbb{C}$ and may have a double pole at $s=1$ and simple poles at $s \in$ $\{2,0,-1,-2,-3, \ldots\}$. In particular, if $\kappa^{\mathrm{t}}=0, \eta_{\mathrm{p}}\left(D_{r}, s\right)$ may have only simple poles at $s \in\{2,1,0,-1,-2,-3, \ldots\}$.

In view of Theorem 6.3, it is natural to define the principal part of the eta invariant of $D_{r}$ by

$$
\eta_{\mathrm{p}}\left(D_{r}\right):=\left.\left(\eta_{\mathrm{p}}\left(D_{r}, s\right)-\frac{r_{0}}{s}\right)\right|_{s=0},
$$

where $r_{0}$ is the residue of the simple pole of $\eta_{\mathrm{p}}\left(D_{r}, s\right)$ at $s=0$. Now let us consider the adiabatic limit of $\eta_{\mathrm{p}}\left(D_{r}\right)$ as $r \rightarrow 0$. For this, we need the following proposition.

Proposition 6.4. As $r \rightarrow 0, \operatorname{Tr}_{\mathrm{p}}\left(D_{r} e^{-t D_{r}^{2}}\right)$ converges to 0 for $t \in(0, \infty)$, and $t^{\frac{3}{2}} \operatorname{Tr}_{\mathrm{p}}\left(D_{r} e^{-t D_{r}^{2}}\right)$ converges to 0 uniformly for $t \in[0,1]$.

Proof. From the expression of $h_{t, r}(\tau)$ in (6.2), we can see

$$
\left|h_{t, r}(\tau)\right| \leqslant C \exp \left(-\frac{r^{2}}{4} t-4 \tau^{2} t\right) \sum_{m \in 2 \mathbb{Z}} e^{-c(m-1)^{2}\left(1+r^{-2}\right) t} \quad \text { for small } r>0,
$$

where $C, c$ are the positive constants that do not depend on $r, \tau$. Hence, the integral

$$
\int_{-\infty}^{\infty} \tau \tanh (\pi \tau) h_{t, r}(\tau) d \tau
$$




\section{P. Loya, S. Moroianu And J. PARK}

vanishes as $r \rightarrow 0$ by the dominated convergence theorem. The same argument holds for the other terms defining $\operatorname{Tr}_{\mathrm{p}}\left(D_{r} e^{-t D_{r}^{2}}\right)$. Hence, $\operatorname{Tr}_{\mathrm{p}}\left(D_{r} e^{-t D_{r}^{2}}\right)$ converges to 0 as $r \rightarrow 0$. The uniform convergence of $t^{\frac{3}{2}} \operatorname{Tr}_{\mathrm{p}}\left(D_{r} e^{-t D_{r}^{2}}\right)$ follows from the following estimate

$$
\left|t^{\frac{3}{2}} \operatorname{Tr}_{\mathrm{p}}\left(D_{r} e^{-t D_{r}^{2}}\right)\right| \leqslant C r^{2} \quad \text { for } t \in[0,1],
$$

which also follows easily from (6.1) and (6.5).

Now we have the following result.

THEOREM 6.5. We have

$$
\lim _{r \rightarrow 0} \eta_{\mathrm{p}}\left(D_{r}\right)=0
$$

Proof. Let us consider

$$
\eta_{\mathrm{p}}\left(D_{r}\right)=\frac{1}{\sqrt{\pi}} \int_{1}^{\infty} t^{-\frac{1}{2}} \operatorname{Tr}_{\mathrm{p}}\left(D_{r} e^{-t D_{r}^{2}}\right) d t+\left.\left(\frac{1}{\Gamma((s+1) / 2)} \int_{0}^{1} t^{(s-1) / 2} \operatorname{Tr}_{\mathrm{p}}\left(D_{r} e^{-t D_{r}^{2}}\right) d t-\frac{r_{0}}{s}\right)\right|_{s=0} .
$$

For the integration over $[1, \infty)$, recalling that $\operatorname{Tr}_{\mathrm{p}}\left(D_{r} e^{-t D_{r}^{2}}\right)$ is exponentially decaying as $t \rightarrow \infty$, it is easy to see that this part vanishes as $r \rightarrow 0$ by Proposition 6.4 and the dominated convergence theorem. By (6.15) the meromorphic extension of the integral $\int_{0}^{1} \cdot d t$ has the following form for $\Re(s) \geqslant-\epsilon$ with small $\epsilon>0$,

$$
\begin{aligned}
\int_{0}^{1} t^{(s-1) / 2} \operatorname{Tr}_{\mathrm{p}}\left(D_{r} e^{-t D_{r}^{2}}\right) d t= & \frac{2 a_{0}}{s-2}+\frac{2 a_{1}}{s-1}-\frac{4 b_{0}}{(s-1)^{2}}+\frac{2 a_{2}}{s} \\
& +\frac{2 a_{3}}{s+1}-\frac{4 b_{1}}{(s+1)^{2}}+\int_{0}^{1} t^{(s-1) / 2} \operatorname{Tr}_{\mathrm{p}}^{*}\left(D_{r} e^{-t D_{r}^{2}}\right) d t
\end{aligned}
$$

where

$$
\operatorname{Tr}_{\mathrm{p}}^{*}\left(D_{r} e^{-t D_{r}^{2}}\right):=\operatorname{Tr}_{\mathrm{p}}\left(D_{r} e^{-t D_{r}^{2}}\right)-a_{0} t^{-\frac{3}{2}}-a_{1} t^{-1}-b_{0} t^{-1} \log t-a_{2} t^{-\frac{1}{2}}-a_{3}-b_{1} \log t .
$$

By Theorem 6.2, all of the coefficients $a_{0}, a_{1}, a_{2}, a_{3}, b_{0}, b_{1}$ (as function of variable $r$ ) vanish as $r \rightarrow 0$. Hence, putting $s=0$ except the term $2 a_{2} / s$, we can see that $-a_{0}-2 a_{1}-4 b_{1}+2 a_{3}-4 b_{1}$ vanishes as $r \rightarrow 0$. For the last integral with $s=0$ also vanishes as $r \rightarrow 0$ since

$$
\left|t^{-\frac{1}{2}} \operatorname{Tr}_{\mathrm{p}}^{*}\left(D_{r} e^{-t D_{r}^{2}}\right)\right| \leqslant C r^{2} \quad \text { for } t \in[0,1]
$$

which follows from (6.16) and the coefficients $a_{0}, a_{1}, a_{2}, a_{3}, b_{0}, b_{1}$ vanish as order of $r^{2}$. This completes the proof.

\section{Eta function of $D_{r}$ : discrete series part}

In this section we study the discrete part of the eta function $\eta_{\mathrm{d}}\left(D_{r}, s\right)$ when $r>0$ is sufficiently small.

First, from Lemma 5.4, let us recall that $h_{t, r}(n)$ is given by $\lambda(n)$ and $\lambda_{ \pm}(n, m)$ and we decompose $\operatorname{Tr}_{\mathrm{d}}\left(D_{r} e^{-t D_{r}^{2}}\right)$ into the corresponding two parts. Then we also have

$$
\eta_{\mathrm{d}}\left(D_{r}, s\right)=\eta_{\mathrm{d}}^{1}\left(D_{r}, s\right)+\eta_{\mathrm{d}}^{2}\left(D_{r}, s\right) \quad \text { for } \Re(s) \gg 0,
$$

where

$$
\eta_{\mathrm{d}}^{1}\left(D_{r}, s\right)=r^{s}(2 g-2+\kappa)\left(-\sum_{k=1}^{\infty} \frac{2(2 k-1)}{\left(2 k-1+r^{2} / 2\right)^{s}}\right)+r^{s} \kappa^{\mathrm{t}}\left(\sum_{k=1}^{\infty} \frac{2}{\left(2 k-1+r^{2} / 2\right)^{s}}\right)
$$


Adiabatic Limit of THE ETA INVARIANT

$$
\begin{aligned}
\eta_{d}^{2}\left(D_{r}, s\right)= & (2 g-2+\kappa)\left(2 \sum_{k=1}^{\infty}(2 k-1) \sum_{\ell \in k+\mathbb{N}} \lambda_{+}(2 k, 2 \ell)^{-s}-\lambda_{-}(2 k, 2 \ell)^{-s}\right) \\
& -\kappa^{\mathrm{t}}\left(2 \sum_{k=1}^{\infty} \sum_{\ell \in k+\mathbb{N}} \lambda_{+}(2 k, 2 \ell)^{-s}-\lambda_{-}(2 k, 2 \ell)^{-s}\right) .
\end{aligned}
$$

Here we used the fact

$$
\operatorname{vol}(\Gamma \backslash G)=2 \pi(2 g-2+\kappa),
$$

where the volume of $\Gamma \backslash G$ is given with respect to the Haar measure in (4.1) (recall that the volume of the circle $K / Z$ is normalized to be 1$)$.

Now we investigate $\eta_{\mathrm{d}}^{1}\left(D_{r}, s\right)$. Let us recall the Hurwitz zeta function

$$
\zeta(s, a)=\sum_{k=0}^{\infty}(k+a)^{-s}
$$

which has a meromorphic extension to the whole $\mathbb{C}$ with a simple pole at $s=1$. If we set

$$
\zeta_{0}(s, a)=\sum_{k=1}^{\infty}(2 k-1+a)^{-s}
$$

then

$$
\zeta_{0}(s, a)=\zeta(s, a)-2^{-s} \zeta\left(s, \frac{a}{2}\right)
$$

By these definitions, for $\Re(s)>2$,

$$
\eta_{\mathrm{d}}^{1}\left(D_{r}, s\right)=2(2-2 g-\kappa) r^{s}\left(\zeta_{0}\left(s-1, \frac{r^{2}}{2}\right)-\frac{r^{2}}{2} \zeta_{0}\left(s, \frac{r^{2}}{2}\right)\right)+2 \kappa^{\mathrm{t}} r^{s} \zeta_{0}\left(s, \frac{r^{2}}{2}\right) .
$$

The right-hand side gives the meromorphic extension of $\eta_{\mathrm{d}}^{1}\left(D_{r}, s\right)$ over $\mathbb{C}$ with simple poles at $s=1,2$. We can also see that $\eta_{\mathrm{d}}^{1}\left(D_{r}, s\right)$ is regular at $s=0$ from this equality. Recalling

$$
\zeta(0, a)=\frac{1}{2}-a, \quad \zeta(-1, a)=-\frac{1}{2}\left(a^{2}-a+\frac{1}{6}\right)
$$

we can see that

$$
\zeta_{0}(0, a)=-\frac{a}{2}, \quad \zeta_{0}(-1, a)=-\frac{1}{4}\left(a^{2}-\frac{1}{3}\right)
$$

Using these, we obtain

$$
\eta_{\mathrm{d}}^{1}\left(D_{r}, 0\right)=(2-2 g-\kappa)\left(\frac{1}{6}+\frac{r^{4}}{8}\right)-\kappa^{\mathrm{t}} \frac{r^{2}}{2} .
$$

Summarizing all of these for $\eta_{\mathrm{d}}^{1}\left(D_{r}, s\right)$, we have the following result.

Proposition 7.1. For a sufficiently small $r>0$, the function $\eta_{\mathrm{d}}^{1}\left(D_{r}, s\right)$, define for $\Re(s)>2$, extends meromorphically to $\mathbb{C}$ and may have simple poles at $s=1,2$. The following equality holds,

$$
\lim _{r \rightarrow 0} \eta_{\mathrm{d}}^{1}\left(D_{r}, 0\right)=\frac{1}{6}(2-2 g-\kappa) .
$$

To obtain the meromorphic extension of $\eta_{\mathrm{d}}^{2}\left(D_{r}, s\right)$ over $\mathbb{C}$, we rewrite this as follows,

$$
\eta_{\mathrm{d}}^{2}\left(D_{r}, s\right)=2(2 g-2+\kappa) r^{s} f_{r}(s)-2 \kappa^{\mathrm{t}} r^{s} g_{r}(s) .
$$


Here

$$
\begin{aligned}
& f_{r}(s)=\sum_{\ell>k \geqslant 1}(2 k-1)\left(\left(q_{r}(k, \ell)-\frac{r^{2}}{2}\right)^{-s}-\left(q_{r}(k, \ell)+\frac{r^{2}}{2}\right)^{-s}\right), \\
& g_{r}(s)=\sum_{\ell>k \geqslant 1}\left(q_{r}(k, \ell)-\frac{r^{2}}{2}\right)^{-s}-\left(q_{r}(k, \ell)+\frac{r^{2}}{2}\right)^{-s}
\end{aligned}
$$

where

$$
q_{r}(k, \ell)=\left((2 \ell-1)^{2}\left(1+r^{2}\right)-r^{2}(2 k-1)^{2}\right)^{\frac{1}{2}} .
$$

Now we put $h_{r}(s)=\sum_{\ell>k \geqslant 1}(2 k-1) q_{r}(k, \ell)^{-s}$ which can be written as

$$
h_{r}(s)=\sum_{k \geqslant 1}(2 k-1)^{1-s} \sum_{\ell>k}(2 \ell-1)^{-s}\left(\frac{1+r^{2}}{(2 k-1)^{2}}-\frac{r^{2}}{(2 \ell-1)^{2}}\right)^{-s / 2} .
$$

From this and the above analysis of $\zeta_{0}(s, 0)$, we can see that $h_{r}(s)$ is holomorphic for $\Re(s)>2$. For the meromorphic extension of $h_{r}(s)$ over $\mathbb{C}$, we use the identity $a^{s}=\exp (s \log (1+(a-1)))$ to obtain

$$
\begin{aligned}
& \left(\frac{1+r^{2}}{(2 k-1)^{2}}-\frac{r^{2}}{(2 \ell-1)^{2}}\right)^{-s / 2} \\
& \quad=1-\frac{s}{2}\left(\frac{1+r^{2}}{(2 k-1)^{2}}-\frac{r^{2}}{(2 \ell-1)^{2}}-1\right)+\frac{s}{4}\left(\frac{1+r^{2}}{(2 k-1)^{2}}-\frac{r^{2}}{(2 \ell-1)^{2}}-1\right)^{2}+\cdots .
\end{aligned}
$$

From this, we can see that $h_{r}(s)$ has the meromorphic extension over $\mathbb{C}$ and may have simple poles at $s=2,1,0,-1, \ldots$ with the residues which are continuous with respect to $r$. Using the following equality

$$
f_{r}(s)=\left(r^{2} s h_{r}(s+1)+r^{6} \frac{s(s+1)(s+2)}{24} h_{r}(s+3)+r^{10} \theta(s, r)\right)
$$

where $\theta(s, r)$ is regular at $s=0$ and is continuous at $r=0$, we can conclude that $f_{r}(s)$ is regular at $s=0$ and the limit of $f_{r}(0)$ as $r \rightarrow 0$ is trivial. In a similar way, we can see that the same conclusion is true for $g_{r}(s)$. By all these facts, we have the following result.

Proposition 7.2. For a sufficiently small $r>0$, the function $\eta_{\mathrm{d}}^{2}\left(D_{r}, s\right)$, defined for $\Re(s)>2$, extends meromorphically to $\mathbb{C}$ and may have simple poles at $s \in\{1,-1,-2,-3, \ldots\}$. The following equality holds,

$$
\lim _{r \rightarrow 0} \eta_{\mathrm{d}}^{2}\left(D_{r}, 0\right)=0
$$

By Propositions 7.1 and 7.2, we can define

$$
\eta_{\mathrm{d}}\left(D_{r}\right):=\left.\eta_{\mathrm{d}}\left(D_{r}, s\right)\right|_{s=0}=\eta_{\mathrm{d}}^{1}\left(D_{r}, 0\right)+\eta_{\mathrm{d}}^{2}\left(D_{r}, 0\right)
$$

and we summarize our results in the following.

TheOREM 7.3. For a sufficiently small $r>0$, the discrete part of the eta function $\eta_{\mathrm{d}}\left(D_{r}, s\right)$, defined for $\Re(s)>2$, extends meromorphically to $\mathbb{C}$ and may have simple poles at $s \in\{2,1,-1,-2,-3, \ldots\}$. Moreover,

$$
\lim _{r \rightarrow 0} \eta_{\mathrm{d}}\left(D_{r}\right)=\frac{1}{6}(2-2 g-\kappa)
$$

\section{ACKNOWLEDGEMENTS}

This work was partly done while the authors visited KIAS and École Polytechnique. The authors would like to express their gratitude to these institutions for their hospitality. Sergiu Moroianu was 


\section{AdiABATIC LIMIT OF THE ETA INVARIANT}

partially supported by a PN-II-ID-PCE-2008-2 contract. The authors also thank the anonymous referee for many helpful comments.

\section{REFERENCES}

AB98 B. Ammann and C. Bär, The Dirac operator on nilmanifolds and collapsing circle bundles, Ann. Global Anal. Geom. 16 (1998), 221-253.

APS75 M. F. Atiyah, V. K. Patodi and I. M. Singer, Spectral asymmetry and Riemannian geometry. I, Math. Proc. Cambridge Philos. Soc. 77 (1975), 43-69.

Bar00 C. Bär, The Dirac operator on hyperbolic manifolds of finite volume, J. Differential Geom. 54 (2000), 439-488.

Bec00 S. Bechtluft-Sachs, The computation of $\eta$-invariants on manifolds with free circle action, J. Funct. Anal. 174 (2000), 251-263.

BF86a J.-M. Bismut and D. S. Freed, The analysis of elliptic families. I. Metrics and connections on determinant bundles, Comm. Math. Phys. 106 (1986a), 159-176.

BF86b J.-M. Bismut and D. S. Freed, The analysis of elliptic families. II. Dirac operators, eta invariants, and the holonomy theorem, Comm. Math. Phys. 107 (1986b), 103-163.

Bor97 A. Borel, Automorphic forms on $\mathrm{SL}_{2}(R)$, Cambridge Tracts in Mathematics, 130 (Cambridge University Press, Cambridge, 1997).

BC89 J. M. Bismut and J. Cheeger, $\eta$-invariants and their adiabatic limits, J. Amer. Math. Soc. 2 (1989), $33-70$.

Che87 J. Cheeger, $\eta$-invariants, the adiabatic approximation and conical singularities. I. The adiabatic approximation, J. Differential Geom. 26 (1987), 175-221.

Dai91 X. Dai, Adiabatic limits, nonmultiplicativity of signature, and Leray spectral sequence, J. Amer. Math. Soc. 4 (1991), 265-321.

DZ95 X. Dai and W. P. Zhang, Circle bundles and the Kreck-Stolz invariant, Trans. Amer. Math. Soc. 347 (1995), 3587-3593.

Don79 H. Donnelly, Asymptotic expansions for the compact quotients of properly discontinuous group actions, Illinois J. Math. 23 (1979), 485-496.

GGP69 I. M. Gel'fand, M. I. Graev and I. I. Pyatetskii-Shapiro, Representation theory and automorphic functions (W. B. Saunders, Philadelphia, PA, 1969).

Hit74 N. Hitchin, Harmonic spinors, Adv. Math. 14 (1974), 1-55.

Hof94 W. Hoffmann, An invariant trace formula for the universal covering group of $\mathrm{SL}(2, \mathbf{R})$, Ann. Global Anal. Geom. 12 (1994), 19-63.

Kna01 A. W. Knapp, Representation theory of semisimple groups. An overview based on examples, Princeton Landmarks in Mathematics (Princeton University Press, Princeton, NJ, 2001).

Lan75 S. Lang, $\mathrm{SL}_{2}(R)$ (Addison-Wesley, Reading, MA, 1975).

LM05 R. Lauter and S. Moroianu, An index formula on manifolds with fibred cusp ends, J. Geom. Anal. 15 (2005), 261-283.

LMP07 E. Leichtnam, R. R. Mazzeo and P. Piazza, The index of Dirac operators on manifolds with fibred boundaries, Bull. Belg. Math. Soc. Simon Stevin 13 (2007), 845-855.

MM90 R. R. Mazzeo and R. B. Melrose, The adiabatic limit, Hodge cohomology and Leray's spectral sequence for a fibration, J. Differential Geom. 31 (1990), 185-213.

MM98 R. R. Mazzeo and R. B. Melrose, Pseudodifferential operators on manifolds with fibred boundaries, Asian J. Math. 2 (1998), 833-866.

Mel93 R. B. Melrose, The Atiyah-Patodi-Singer index theorem, Research Notes in Mathematics, 4 (A K Peters, Wellesley, MA, 1993).

Nic98 Nicolaescu L. I., Adiabatic limits of the Seiberg-Witten equations on Seifert manifolds, Comm. Anal. Geom. 6 (1998), 331-392. 


\section{ADIABATIC LIMIT OF THE ETA INVARIANT}

Nic99 Nicolaescu L. I. Eta invariants of Dirac operators on circle bundles over Riemann surfaces and virtual dimensions of finite energy Seiberg-Witten moduli spaces, Israel J. Math. 114 (1999), 61-123.

Par05 J. Park, Eta invariants and regularized determinants for odd dimensional hyperbolic manifolds with cusps, Amer. J. Math. 127 (2005), 493-534.

SS87 J. Seade and B. Steer, A note on the eta function for quotients of $\mathrm{PSL}_{2}(R)$ by co-compact Fuchsian groups, Topology 26 (1987), 79-91.

Ste89 M. Stern, $L^{2}$-index theorems on locally symmetric spaces, Invent. Math. 96 (1989), 231-282.

Vai01 B. Vaillant, Index and spectral theory for manifolds with generalized fibred cusps, Dissertation, Rheinische Friedrich-Wilhelms-Universitat Bonn, Bonn (2001).

Wit85 E. Witten, Global gravitational anomalies, Comm. Math. Phys. 100 (1985), 197-229.

Woj K. P. Wojciechowski, Witten's holonomy theorem in the non-loop case, unpublished note.

Zha94 W. P. Zhang, Circle bundles, adiabatic limits of $\eta$-invariants and Rokhlin congruences, Ann. Inst. Fourier (Grenoble) 44 (1994), 249-270.

Paul Loya paul@math.binghamton.edu

Department of Mathematics, Binghamton University, Binghamton, NY 13902, USA

Sergiu Moroianu moroianu@alum.mit.edu

Institutul de Matematică al Academiei Române, PO Box 1-764, RO-014700 Bucharest, Romania

Current address: Şcoala Normală Superioară Bucharest, Calea Grivitei 21, Bucharest, Romania

Jinsung Park jinsung@kias.re.kr

School of Mathematics, Korea Institute for Advanced Study, 207-43, Cheongnyangni 2-dong,

Dongdaemun-gu, Seoul 130-722, Korea 